Center for

Mathematical Economics

Working Papers

\title{
493
}

December 2013

\section{Radner Equilibria under Ambiguous Volatility}

Patrick Beißner

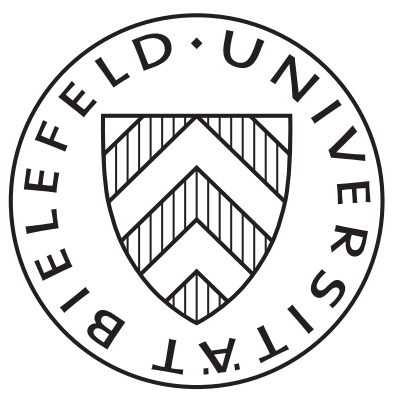




\title{
Radner Equilibria under Ambiguous Volatility
}

\author{
Patrick Beißner*
}

December 92013

\begin{abstract}
The present paper considers a class of general equilibrium economies when the primitive uncertainty model features uncertainty about continuous-time volatility. This requires a set of mutually singular priors, which do not share the same null sets. For this setting we introduce an appropriate commodity space and the dual of linear and continuous price systems.

All agents in the economy are heterogeneous in their preference for uncertainty. Each utility functional is of variational type. The existence of equilibrium is approached by a generalized excess utility fixed point argument.

Such Arrow-Debreu allocations can be implemented into a Radner economy with continuous-time trading. Effective completeness of the market spaces alters to an endogenous property. Only mean unambiguous claims equivalently satisfying the classical martingale representation property build the marketed space.
\end{abstract}

Key words and phrases: Knightian uncertainty, variational preferences, general equilibrium, mutually singular priors, dynamic consistency, volatility uncertainty, excess utility map, gross substitutes, risk adjusted priors, sublinear-expectation, Radner implementation, incomplete markets

JEL subject classification: G10, D50, C62

\section{Introduction}

Ever since the pioneering general theory of competitive markets, the extension to a dynamic equilibrium has served as an initial position for a neoclassical intertemporal asset pricing theory.

Most models of an Arrow-Debreu economy in continuous time assume an underlying and a priori given probabilistic structure. We replace this all-encompassing and basic assumption with a set of pairwise mutually singular probability measures (priors) $\mathcal{P}$. Our main focus is concerned with models where the volatility of the

${ }^{*}$ I thank Frank Riedel for many fruitful discussions. Center for Mathematical Economics Bielefeld University, 33501 Bielefeld, Germany. Email: pbeissne@math.uni - bielefeld.de. Financial support through the German Research Foundation (DFG) and the IGK "Stochastics and Real World Models" Beijing-Bielefeld are gratefully acknowledged. 
state variable is uncertain or ambiguous. This can only be accomplished through one such set. Furthermore, we aim to analyze the interrelation between volatility uncertainty and incomplete markets. In contrast to the situation of mutually equivalent priors $1^{1}$ a new feature emerges about the states of the world:

Certainty about the true prior automatically determines states which cannot occur. A different situation arises when certainty is limited to the knowledge that the true prior is contained in $\mathcal{P}$. This shrinks the set of impossible states and reasonable contingent claims.

The existing literature, when dealing with potentially complete markets, has established a standard way to construct a financial market equilibrium. Here Duffie and Huang (1985) may be regarded as the seminal paper that explores the idea in Kreps (1982), about implementing an Arrow-Debreu allocation into a so called Radner (1972) economy. This is achieved via continuous trading of long-lived securities. A major tool for spanning the complete market of Arrow-Debreu securities is the concept of a martingale generator, which reduces in a Brownian setting to the classical martingale representation theorem. $2^{2}$ However, in the present setup the concept of martingale multiplicity as an integer valued measure for the dimension of uncertainty is imprecise. An additional component in the martingale representation suggests, instead, a measure with fraction number values.

This paper establishes the existence of a Radner equilibrium with an endogenously incomplete financial market. The starting point is a heterogeneous agent ArrowDebreu economy with ambiguity averse agents, where the objective uncertainty is given by the set of priors $\mathcal{P}$. Similarly to representative agent economy in Epstein and Wang (1994) we observe the indeterminacy in the effective equilibrium priors of the price system, as output data of this intermediate economy. As a result, only special Arrow-Debreu equilibrium allocation can be implemented into a Radner economy, and we observe an incomplete market equilibrium. The endogenous indeterminacy of the Arrow-Debreu equilibrium price system determines the degree and structure of the incompleteness of the implementing financial market.

In the present Radner economy, each agent has to find trading strategies of buying and selling traded claims in order to maximize her utility on net trades when volatility uncertainty of the state variable is present. This is achieved in terms of a suitable dynamic conditional sublinear expectation $X \mapsto \mathbb{E}_{t}^{\mathcal{Q}_{E}}[X] !^{3}$ The set $\mathcal{Q}_{E}$

\footnotetext{
${ }^{1}$ Ambiguity or Knightian uncertainty in continuous time is often modeled by the so called drift uncertainty. Here, the probabilities must be equivalent to each other. Such a description is not appropriate when the volatility is the object which carries the uncertainty. See Chen and Epstein (2002) for a formulation of such preferences via a backward stochastic differential equation (Backward-SDE) and Beißner (2011) for the related existence of general equilibrium.

${ }^{2}$ The notion of martingale multiplicity works in a separable framework, so that an orthogonalization procedure counts the dimension of uncertainty.

${ }^{3}$ At this point the assumed weak compactness and stability under pasting of $\mathcal{P}$ play an essential role for the construction of a universal random variable being under each prior simultaneously the conditional expectation. When the set of priors is mutually equivalent, this property is nothing else as the dynamic consistency of conditional expectation. In the volatility uncertainty framework stability under pasting is a stronger condition, see Nutz and Soner (2012). A key feature of this conditional expectation is the semigroup property $\mathbb{E}_{s} \circ \mathbb{E}_{t}=\mathbb{E}_{s}$ for $s \leq t$, which implies the Law of Iterated Expectation.
} 
refers to all equilibrium price measures, given an equilibrium allocation. In the classical uncertainty model with only one prior, the linear risk-adjusted expectation operator is related to the unique equilibrium price measure.

As demonstrated in the finite state case, Mukerji and Tallon (2001) discuss ambiguity aversion as a source for incompleteness in financial markets. Beyond the related marketed space, a kind of collective portfolio inertia results. In essence, the market-clearing condition in the Radner equilibrium is in action. The role of the financial market as a mechanism to change the shape of income streams is accomplished only partially. Nevertheless, this fits into the arguments by Dow and da Costa Werlang (1992), where inertia for a single agent in a partial equilibrium is detected. In a different setting, De Castro and Chateauneuf (2011) observe similar results on unambiguous trade with unambiguous aggregate endowment.

As argued in Anderson and Raimondo (2008), the candidate equilibrium price process is often assumed to be dynamically complete. Quite frequently this assumption is encoded in the exogenous volatility model of the candidate equilibrium price process ${ }^{4}$ In this regard, our model differs in terms of an intrinsic incompleteness due to the volatility uncertainty and the appearance of ambiguous net trades. As such, the size and structure of the marketed space is the result of Arrow-Debreu equilibrium.

\section{Martingales and Dynamic Spanning}

The relationship between martingale multiplicity and dynamical spanning of the commodity space is an economically meaningful corollary of the martingale representation. In the case of Brownian noise a square integrable random variable $X$ can be represented in terms of a stochastic integral:

$$
X=\mathrm{E}^{P}[X]+\int_{0}^{T} \theta_{s} \mathrm{~d} B_{s}
$$

This result is strongly related to the completeness of the financial market. Loosely speaking, in our mutually singular prior framework, a number representing the dimensions of uncertainty does not exist. In essence, this is caused by the more evolved martingale representation theorem. Similarly to the classical Doob-Meyer decomposition for a submartingale, the representation of martingales under a sublinear expectation sustain an additional monotone compensation term:

$$
X=\mathbb{E}^{\mathcal{P}}[X]+\int_{0}^{T} \theta_{s} \mathrm{~d} B_{s}-K_{T}
$$

Only a closed subspace of the present commodity space $L^{1}(\mathcal{P})$ allows for the classical replication of a possible consumption profile $X: \Omega \rightarrow \mathbb{R}$. In this case the compensation term $\left(K_{t}\right)$ equals zero. Such random variables are mean unambiguous, i.e. the expectation value of the claim is the same under each prior. At this abstract stage, we can already presume some implications for incompleteness in the involved market structure, see Remark 3.1.

\section{The uncertainty model and the economy}

\footnotetext{
${ }^{4}$ See for instance Duffie and Zame (1989) and Karatzas, Lehoczky, and Shreve (1990).
} 
We consider a measurable space $(\Omega, \mathcal{F})$ and fix a set of the probability measures $\mathcal{P}$. In general, three cases of relationships between priors in $\mathcal{P}$ are possible. As described at the beginning of the introduction, two priors maybe mutually singular. This implies a disjoint support of these measures. The second possibility is a mixture. In this case, two priors may be equivalent on a sub $\sigma$-field and mutually singular on a complementary sub $\sigma$-field. The last case, which does not appear, is mutual equivalence of measures.

In principle, this modeling can describe a set of different probability assessments related to the states of the world $\omega \in \Omega$, so that different possible shapes of the intrinsic volatility may appear. Sure statements concerning random variables in this uncertainty setting cannot be reflected as almost sure events under only one prior $P \in \mathcal{P}$. In this context, arguments are based on $\mathcal{P}$-quasi sure analysis, which takes every prior into account simultaneously. Here, a reasonable consumption profile $X: \Omega \rightarrow \mathbb{R}$ should have a finite first moment. Thus, our commodity space $L^{1}(\mathcal{P})$ consists of random variables with a finite expectation for all $P \in \mathcal{P}$.5 $^{5}$ Based on this sublinear expectation, we can define a norm $c_{1, \mathcal{P}}$ such that the space of consumption profiles becomes a Banach space. The positive cone of $L^{1}(\mathcal{P})$, given by random variables satisfying $X \geq 0 \mathcal{P}$-quasi surely, induces an appropriate order structure. Having the commodity space fixed, we introduce the corresponding topological dual space. This space consists of continuous and linear functionals, which are the candidate price systems. Similarly to the single prior case a generalized Radon-Nikodym density result, representing these price functionals, becomes available. In essence, we can represent every linear and $c_{1, \mathcal{P}}$-continuous functional by a measure $\mu$ such that $\mathrm{d} \mu=\psi \mathrm{d} P$, where $P \in \mathcal{P}$ and $\psi \in L^{\infty}(P)$. This allows us to approach the existence of equilibria via a modified excess utility mapping.

With the given commodity-price duality, we introduce a class of preference relations for the agents in the economy. In the seminal paper by Gilboa and Schmeidler (1989), the well-known maxmin preferences are axiomatized, and account for ambiguity aversion. Later Hansen and Sargent (2001) generalize this concept by introducing an entropy based penalty term for the priors under consideration 6 In our economy, agents are described by variational preferences. Maccheroni, Marinacci, and Rustichini (2006) introduce and axiomatize variational preferences, a robust version of the expected utility in the form

$$
U(X)=\min _{P \in \mathcal{P}} \mathrm{E}^{P}[u(X)]+\mathfrak{c}(P),
$$

where the minimum is taken by a whole class of possible probabilistic views of conceivable scenarios. The functional $\mathfrak{c}: \mathcal{P} \rightarrow \mathbb{R}$ penalizes each prior with a different weight. We show that natural properties, such as concavity and upper semicontinuity are imposed when natural conditions on the primitives. When the penalty term is linear even $c_{1, \mathcal{P}}$-continuity can be shown. Moreover, we fully describe the superdifferential of such a utility functional, as in Rigotti and Shannon (2012) for the finite state case.

The economy consists of $I \in \mathbb{N}$ agents, equipped with variational preferences on

${ }^{5}$ For instance, for each $P \in \mathcal{P}$, the commodity space satisfies $L^{1}(\mathcal{P}) \subset L^{1}(\Omega, \mathcal{F}, P)$.

${ }^{6}$ Note that in their model the set of priors are mutually equivalent. 
the positive cone of the commodity space $L^{1}(\mathcal{P})$. The existence of equilibrium is achieved by a modified Negishi method. In the first step we prove the existence of Pareto optimal allocations $5^{7}$ The modification of the excess utility relies on multiple priors, which are now explicit arguments of the excess utility map. 8

In the last part, we implement the net trades of the equilibrium allocation into a Radner type economy. This is achieved via the previously mentioned martingale representation. The implementability of the Arrow-Debreu Equilibrium is limited by the linear price system.

\section{Related Literature}

In the standard single prior Arrow-Debreu setting with expected utility, market prices are directly affected via individual marginal rates of substitution for state contingent commodity bundles (See Martins-da Rocha and Riedel (2010) for a general overview of issues concerning issues the existence of equilibria.) In the simplest version of this model, equilibrium price systems are given by marginal utility weights that can result into risk-neutral probabilities. Continuous-time models and dynamic Arrow-Radner equilibria are treated in Duffie and Huang (1985) and Dana and Pontier (1992). A unique Radner equilibrium is observed in Karatzas, Lehoczky, and Shreve (1990). This approach is based on a representative agent, see Huang (1987). We also refer to Hugonnier, Malamud, and Trubowitz (2012) and Herzberg and Riedel (2013) for a recent discussion of endogenous completeness in continuoustime finance models.

Existence of equilibria in incomplete markets for a finite state space is well developed, starting with the seminal paper by Duffie and Shafer $(1985)$. For an overview we refer the reader to Magill and Quinzii (2002). In Basak and Cuoco (1998), restricted market participation is modeled as a source of market incompleteness. As a consequence, Pareto weights are stochastic.

When the uncertainty is given by an undominated multiple-prior setting, considerations of heterogeneous agent economies are treated only for a finite state space, see for instance Dana (2004) and Dana (2002). In Dana and Le Van (2010) no-arbitrage conditions are associated with a risk adjusted set of priors. Rigotti and Shannon (2012) discuss market implications of ambiguity and feature generic determinacy of general equilibrium.

Ravanelli and Svindland (2013) consider efficient allocation with variational preference when the uncertainty is given by a set of equivalent probability measures. In this case, it is possible to start with a reference probability space.

Representative agent economies for the infinite state and discrete time case can be found in Epstein and Wang (1994), where a modification of Lucas' asset pricing model is established in terms of a Choquet expected utility introduced in Chateauneuf (1991). Very recent research by Epstein and Ji (2013a) provide a discussion of the continuous-time case and the notion of sequential trade equilibria with a single agent.

\footnotetext{
${ }^{7}$ Here, the topological lattice properties of the commodity space ease the proof for the existence of an optimal allocation.

${ }^{8}$ Several technical difficulties motivate this change. A particular problem is that the price space is not directly related to a state price density as in the traditional Lebesgue space setting when there is only one prior $P$.
} 
This paper is organized as follows. Section 2 illustrates the implications of the uncertainty model in the case of finitely many states or priors. In Section 3 we introduce the commodity space and the price space. Moreover, we introduce the variational utility functional and discuss its properties. In Section 4, we show the existence of Pareto optimal allocations. Afterwards we establish the existence of equilibrium and the Radner implementation. The appendix collects the details and proofs.

\section{Simple Economies under Singular Priors}

For perspective, we give an outline about the implication of maxmin preferences when there are finitely many states of world $\Omega=\left\{\omega_{1}, \ldots, \omega_{n}\right\}$. As we will see, the worst case expected utility with a partially disjoint support of possible priors emerges in the form of a Leontief-type utility. In the first subsection, we illustrate the implication in a concrete two agent economy with two priors $\mathcal{P}=\left\{P_{1}, P_{2}\right\}$ on $\Omega$ that are neither singular nor equivalent. Then, we move to the setting with the state space found in Sections 3 and 4 and foreclose some results formulated therein. Two priors $P_{1}, P_{2} \in \stackrel{\circ}{\Delta}_{n}$, the interior of the simplex of probability measures, are always equivalent. Two priors are singular if their supports are disjoint.

\subsection{The Finite State Case}

In order to illustrate the main point with a concrete example, consider an economy with two agents $i=1,2$ and $n=6$ states of the world at time $T>0$. The uncertainty is given by two measures represented by $P_{1}=\left(0,0, \frac{1}{4}, \frac{1}{4}, \frac{1}{4}, \frac{1}{4}\right)$ and $P_{2}=$ $\left(\frac{1}{4}, \frac{1}{4}, \frac{1}{4}, \frac{1}{4}, 0,0\right)$, see Figure 1.9 It is unknown which prior is the correct, although

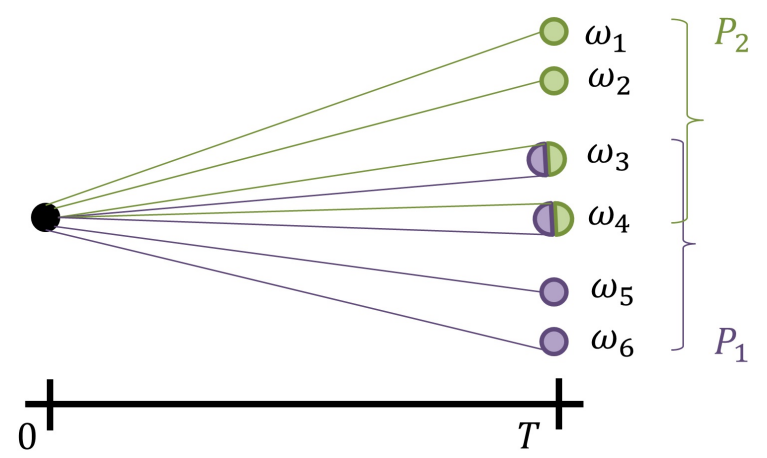

Figure 1: Non-Equivalent and Non-Singular Priors

each prior determines different states of the world. Each agent is ambiguity averse on $\mathcal{P}=\left\{P_{1}, P_{2}\right\}$ with maxmin preferences represented in terms of $U^{i}: \mathbb{R}_{+}^{6} \rightarrow \mathbb{R}$

\footnotetext{
${ }^{9}$ In the volatility uncertainty setting, such priors occur when the volatility is in agreement up to some time $t>0$ and then differs.
} 
given by

$$
\begin{aligned}
U^{i}(X) & =\min _{P \in \mathcal{P}} E^{P}\left[a^{i} \ln (X)\right] \\
& =\frac{a_{i}}{4}\left(\ln \left(X_{\omega_{3}} \cdot X_{\omega_{4}}\right)+\min \left(\ln \left(X_{\omega_{1}} \cdot X_{\omega_{2}}\right), \ln \left(X_{\omega_{5}} \cdot X_{\omega_{6}}\right)\right)\right) .
\end{aligned}
$$

The endowments are given by $e^{1}=(1,1,2,1,3,3)$ and $e^{2}=(2,2,1,2,1,1)$, where the prior-dependent endowment is denoted by $e^{i}(P)$, for instance we have $e^{1}\left(P_{1}\right)=(1,1,2,1)$. Due to the singularity in the events $\left\{\omega_{1}, \omega_{2}\right\}$ and $\left\{\omega_{5}, \omega_{6}\right\}$, the utility structure has a Leontief flavor in these states. This means for instance, the indifference curve with respect to payoffs in the events $\left\{\omega_{1}, \omega_{2}\right\}$ and $\left\{\omega_{5}, \omega_{6}\right\}$ are $L$-shaped. This is illustrated in the Edgeworth boxes of Figure 2.

After some calculations, we have an equilibrium price system $\Pi(\cdot)=\langle\cdot, p\rangle$, with $p \in \mathbb{R}_{+}^{6}$ such that $\left(p_{5}, p_{6}\right)=0$ must hold. This follows from the $L$-shaped indifference curve and $\left(e_{\omega_{1}}, e_{\omega_{2}}\right)>\left(e_{\omega_{5}}, e_{\omega_{6}}\right)$. The price system has the same support as $P_{2}$. This can be infered from the first order conditions, since each agent has $P_{2}$ as the minimizing (effective) prior of her maxmin utilities. The non-unique equilibrium allocation $\left(\bar{X}^{1}, \bar{X}^{2}\right) \in[\underline{X}, \bar{X}]$ lies on the orange line segment of Figure $2(\mathrm{~b})$. Arrow securities of state $\omega_{5}$ and $\omega_{6}$ are for free, so that a feasible retrade on the order interval $[\underline{X}, \bar{X}]$ leaves the utility unaffected. On the other hand, consump-
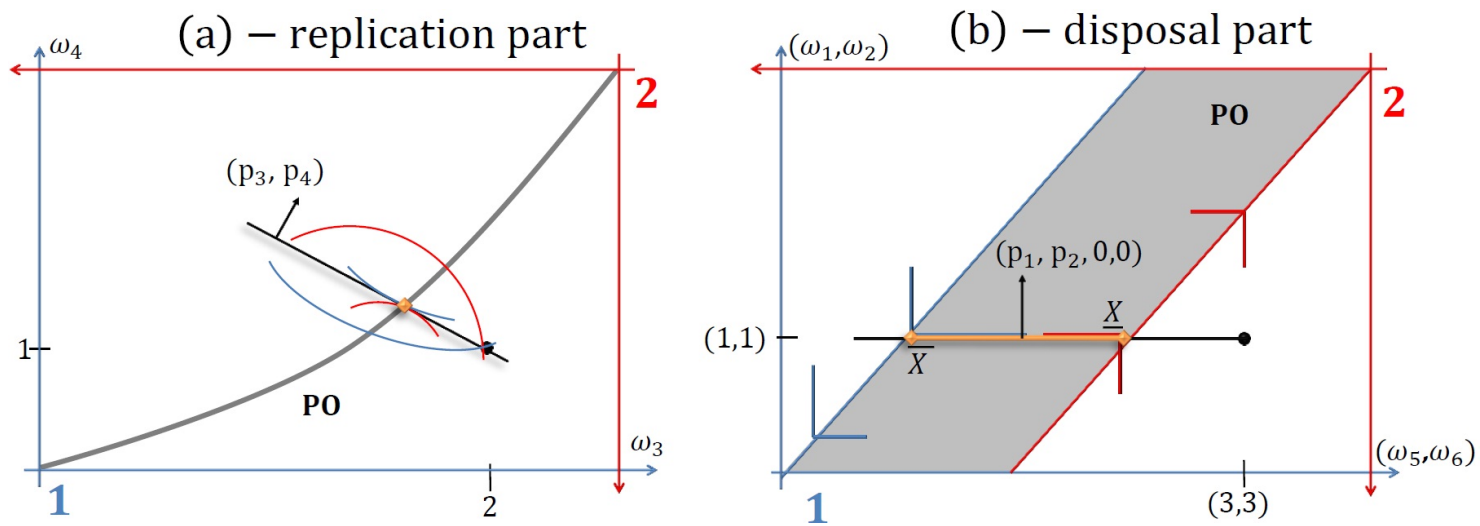

Figure 2: Edgeworth boxes via Leontief-type utility

tion in state $\omega_{3}$ and $\omega_{4}$ behaves as in the expected utility setting with one prior. Specifically, the consumption is prior independent. This can be seen in the explicit description of $U^{i}(X)$ above and Figure 2 (a). In Subection 3.1.2 we consider the analog space of unambiguous contingent claims denoted by $\mathrm{M}[\mathcal{P}]$. However, trade outside of $\mathrm{M}[\mathcal{P}]$ is possible.

\subsubsection{The Modified Negishi-Approach}

Continuing with the setting of the last paragraph, we illustrate how the existence of an equilibrium can be shown. To do so, we consider the first order condition

$$
\alpha^{i} \nabla U^{i}\left(\bar{X}^{i}\right)=\alpha_{i}\left(\frac{a_{i} \cdot P_{2}\left(\left\{\omega_{1}\right\}\right)}{\bar{X}_{\omega_{1}}^{i}}, \ldots, \frac{a_{i} \cdot P_{2}\left(\left\{\omega_{4}\right\}\right)}{\bar{X}_{\omega_{4}}^{i}}, 0,0\right)=\langle p, \cdot\rangle, \quad i=1,2 .
$$


From this characterization of the Pareto optimal (PO) allocation, we denote the set of common effective priors under the efficient allocation by $\mathbb{P}(\alpha)$. The restriction to concentrate on linear prices leads to a price system $\langle p, \cdot\rangle=\mathrm{E}^{P_{2}}[\psi \cdot]$ having an endogenous support $\left\{\omega_{1}, \ldots, \omega_{4}\right\}$. As such the representation as a sole random variable fails.

We illustrate how the Negishi method applies to show the $P_{2}$ almost sure unique equilibrium, so that the indeterminacy of the equilibrium allocation is outside the support of $P_{2}$. Let us consider the utility possibility set in Figure 3. The utility

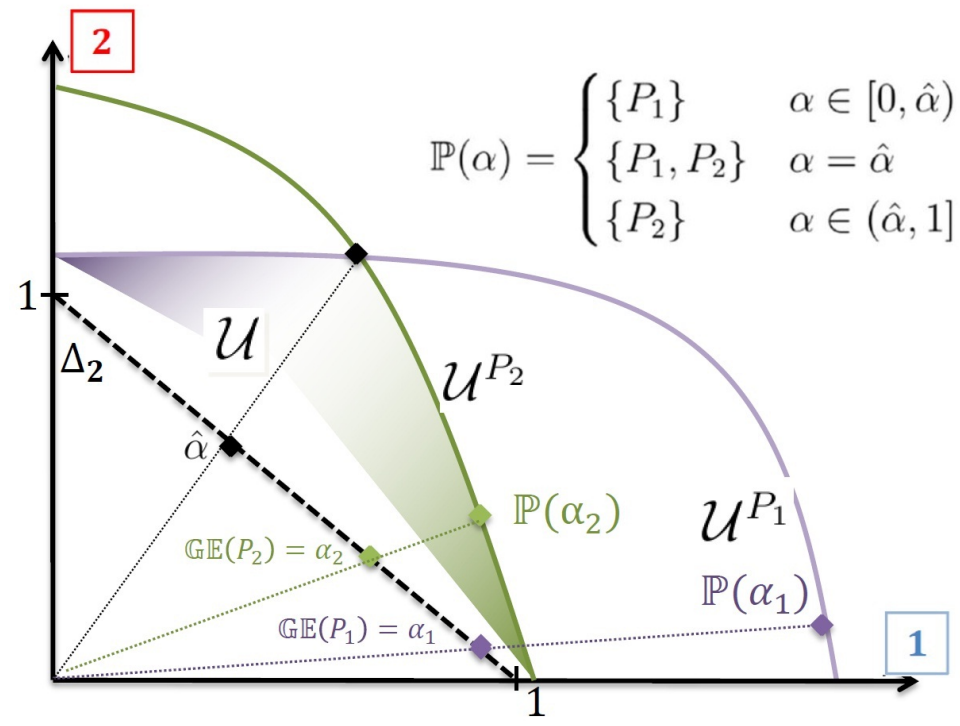

Figure 3: Utility possibility set under $\left\{P_{1}, P_{2}\right\}=\mathcal{P}$

possibility set (UPS) for the economy $\mathcal{E}^{P}$ with expected log utility agents under $P \in \mathcal{P}$ is denoted by $\mathcal{U}^{P}$. Clearly, each $\mathcal{E}^{P}$ induces a unique equilibrium weight denoted by $\alpha=\mathbb{G} \mathbb{E}(P)$. On the other hand each $\alpha \in \Delta_{2}$ induces a representative agent $U_{\alpha}$, whose effective prior is denoted by $P \in \mathbb{P}(\alpha)$. The UPS of the original economy with multiple priors is then given by $\mathcal{U}=\mathcal{U}^{P_{1}} \cap \mathcal{U}^{P_{2}}$. Moreover, we have $P_{2} \in \mathbb{P}\left(\alpha_{2}\right)$. While $P_{1} \notin \mathbb{P}\left(\alpha_{1}\right)$ is not an effective prior for $\mathcal{U}^{P_{1}}$ and therefore contradicts the first order conditions with respect to the $\alpha_{1}$-efficient allocation. This illustrates how the Negishi approach with a von Neumann-Morgenstern utility still applies under the correct prior of the maxmin preferences, as explained in the following.

An equilibrium has to satisfy two conditions. On the one hand, the prior $P_{2}=P^{*}$ as a component of the price system must be effective for the representative agent, i.e. $P^{*} \in \mathbb{P}\left(\alpha^{*}\right)$. On the other hand the weighting $\alpha^{*}$ of the representative agent under $P^{*}$ must be the correct equilibrium weight denoted by $\mathbb{G} \mathbb{E}\left(P^{*}\right)=\alpha^{*}$. These two conditions can be condensed in a fixed point of a composited correspondence, i.e. $P^{*} \in \mathbb{P} \circ \mathbb{G} \mathbb{E}\left(P^{*}\right)$. This observation will lead to a proof method for the existence of an equilibrium, also in the volatility uncertainty setting. Moreover, as a byproduct, we observe structural properties more directly. 


\subsection{The Infinite State Case}

One special property of every finite dimensional commodity space $L$ is the equivalence of every two arbitrary norms $\|\cdot\|_{i}: L \rightarrow \mathbb{R}_{+}, i=1,2$, with this in mind, we move to the infinite (and uncountable) state space $\Omega$, consisting of continuous paths $\omega:[0, T] \rightarrow \mathbb{R}$, equipped with the usual Borel $\sigma$-algebra $\mathcal{B}(\Omega)=\mathcal{F}$. Let us consider two mutually singular priors $\mathcal{P}=\left\{P_{1}, P_{2}\right\}$ on $(\Omega, \mathcal{F})$ as the uncertainty model. In Section 3 we describe this in more detail.

Let the endowment $e_{i}$ of each agent $i=1,2$ depend on the prior. So that we have $e_{i}=\left(e_{i}^{P_{1}}, e_{i}^{P_{2}}\right) \in L^{2}\left(P_{1}\right) \times L^{2}\left(P_{2}\right)$, where $L^{2}(P)=L^{2}(\Omega, \mathcal{F}, P)$ is the usual Lebesgue space of integrable random variables equipped with a standard norm $\|x\|_{L^{2}(P)}=\mathrm{E}^{P}\left[|x|^{2}\right]^{1 / 2}$. Since both priors are possible, it is reasona-ble to consider endowments satisfying $c_{2, \mathcal{P}}\left(e_{i}\right)=\max \left\{\left\|e_{i}^{P}\right\|_{L^{2}(P)}, P \in \mathcal{P}\right\}<\infty$. The finiteness condition under the $c_{2, \mathcal{P}}$-norm corresponds to the space $L^{2}(\mathcal{P})$, being with each $P \in \mathcal{P}$ a strict sub space of $L^{2}(P)$.

A standard price system $\Pi: L^{2}(\mathcal{P}) \rightarrow \mathbb{R}$ for equilibria in infinite dimensional commodity space is linear and continuous in the topology of the underlying commodity space. As we will present in Section 3.1 the related price dual space of $L^{2}(\mathcal{P})$, denoted by $L^{2}(\mathcal{P})^{*}$, is strictly larger than $L^{2}(P)^{*}, P \in \mathcal{P}$, due to the stronger $c_{2, \mathcal{P}}$-norm. We have the following sequence of inclusions:

$$
L^{2}(\mathcal{P}) \subset L^{2}(P) \cong L^{2}(P)^{*} \subset L^{2}(\mathcal{P})^{*}, \quad P \in \mathcal{P}
$$

Again, each agent has maxmin utility $U_{i}(X)=a_{i} \min \left(\mathrm{E}^{P_{1}}[\ln (X)], \mathrm{E}^{P_{2}}[\ln (X)]\right)$, defined on the positive cone $L^{2}(\mathcal{P})_{+}$. Let $\left(\bar{X}_{1}, \bar{X}_{2}\right)$ be an equilibrium allocation of the economy $\mathcal{E}=\left\{L^{2}(\mathcal{P}), U_{i}, e_{i}\right\}_{i=1,2}$ such that $\bar{X}_{1}^{P}+\bar{X}_{2}^{P}=e^{P}$ and $\bar{X}_{1}^{P}=\bar{X}_{1} P$-a.s. holds under every $P \in \mathcal{P}$.

Now, consider the situation when $U_{i}\left(\bar{X}_{i}\right)=a_{i} \mathrm{E}^{P_{2}}\left[\ln \left(\bar{X}_{i}\right)\right] \neq a_{i} \mathrm{E}^{P_{1}}\left[\ln \left(\bar{X}_{i}\right)\right]$ for each $i=1,2$. The supergradients of $U_{i}$ at a consumption bundle in $L^{2}(\mathcal{P})_{+}$lie in the dual $L^{2}(\mathcal{P})^{*}$. The first order condition to characterize a Pareto optimal allocation gives us

$$
\alpha_{1} \cdot \nabla U_{1}\left(\bar{X}_{1}\right)=\mu_{1}=\mu_{2}=\left(1-\alpha_{1}\right) \cdot \nabla U_{2}\left(\bar{X}_{2}\right) \text {, where } \mathrm{d} \mu_{i}=\alpha_{i} \cdot u_{i}^{\prime}\left(\bar{X}_{i}\right) \mathrm{d} P_{2} .
$$

In comparison to the traditional general equilibrium theory the equilibrium pricing measure $Q=\frac{\mu}{|\mu|}$ cannot contain all the information about the uncertainty model. Although $Q$ completely represents the linear and continuous price system $\Pi(\cdot)=\mathrm{E}^{Q}[\cdot]$, it is decoupled from the non-effective prior (relatively to the equilibrium allocation) $P_{1}$. Note, that this conceptual observation is consistent with the finite state case in Subsection 2.1. These informal computations are condensed in the following observation:

Let $\left(p,\left(\bar{X}_{i}\right)\right)$ be an equilibrium in $\left\{L^{2}\left(P_{2}\right), U_{i}, e_{i}\right\}_{i=1}^{2}$ then an equilibrium for $\left\{L^{2}\left(P_{1}\right) \times L^{2}\left(P_{2}\right), U_{i}, e_{i}\right\}_{i=1}^{2}$ is given by $\left((0, p),\left(\bar{X}_{1}^{P_{1}}, \bar{X}_{1}^{P_{2}}\right),\left(\bar{X}_{2}^{P_{1}}, \bar{X}_{2}^{P_{2}}\right)\right)$.

In contrast to a standard finance model with an underlying probability space $(\Omega, \mathcal{F}, P)$, the equilibrium price system $\Pi \in L^{2}(\mathcal{P})^{*}$ no longer carries the information of all null sets. This has direct implications for the related concepts of asset pricing, such as arbitrage, equivalent martingale measures and stochastic discount factors. 


\section{The Primitives of the Economy}

Let us start with the underlying uncertainty model. We consider scenarios, represented by probabilistic priors, which do not share the same null sets. As such, it is not appropriate to assume the existence of a given reference probability measure. Concerning the construction of priors, our method needs some structure on the state space.

Let $\Omega$ be the set of all possible states of the world. A state is an exogenous sequence of circumstances from time 0 to time $T$ which are relevant to the economy. We assume $\Omega=\left\{\omega \in C([0, T] ; \mathbb{R}): \omega_{0}=0\right\}$ to be the canonical space of continuous sample paths starting in zero and endowed with the uniform topology ${ }^{10}$ The $\sigma$-field of events is given by the Borel $\sigma$-field of $\Omega$, called $\mathcal{F}=\mathcal{B}(\Omega)$. Let $\mathcal{M}_{1}(\Omega)$ be the set of all probability measure on $(\Omega, \mathcal{F})$.

Now, we construct a set of priors on the measurable space $(\Omega, \mathcal{F})$. The canonical process $B_{t}(\omega)=\omega_{t}$ is a Brownian motion under the Wiener measure $P_{0}{ }^{11}$ We denote by $\mathbb{F}^{o}=\left\{\mathcal{F}_{t}^{o}\right\}_{t \in[0, T]}$, with $\mathcal{F}_{t}^{o}=\sigma\left(B_{s}, s \in[0, t]\right)$ the raw filtration of the canonical process $B$. The strong formulation of volatility uncertainty is based upon martingale laws in terms of stochastic integrals:

$$
P^{a}:=P_{0} \circ\left(X^{a}\right)^{-1}, \quad \text { where } X_{t}^{a}=\int_{0}^{t} \sqrt{a_{s}} \mathrm{~d} B_{s}, \quad t \in[0, T] .
$$

The stochastic integral $X^{a}$ is the classical Itô integral under $P_{0}$. The process $a=\left(a_{t}\right)_{t \in[0, T]}$ is $\mathbb{F}^{o}$-adapted and has a finite first moment. Probability measures generated in this way are denoted by $\mathcal{P}_{S}$, referring to the strong formulation of volatility uncertainty.

Assumption 1 The uncertainty of each agent is generated by a convex set $\mathcal{D}$ of processes, such that the set of priors is weakly compac ${ }^{12}$ and given by

$$
\mathcal{P}=\left\{P^{a} \in \mathcal{P}_{S}: a \in \mathcal{D}\right\} .
$$

Recall that, the volatility of a stochastic integral $X^{a}=\int \sqrt{a} \mathrm{~d} B$ is given by the quadratic variation $\left\langle X^{a}\right\rangle_{t}=\int_{0}^{t} a_{s} \mathrm{~d} s$. As such, by construction the volatility uncertainty is encoded in the quadratic variation. The mutual singularity of priors is an intrinsic and natural property in the continuous-time setting. For instance, $P^{a}\left(\left\langle B_{T}\right\rangle=T\right)=0 \neq 1=P_{0}\left(\left\langle B_{T}\right\rangle=T\right)$ may appear, for some constant $a \neq 1$.

In order to address this fact, we need to modify the notion of a sure event. To do so, we say a property holds $\mathcal{P}$-quasi surely $(\mathcal{P}$-q.s.) if it holds outside a $\mathcal{P}$-polar set. Such sets have zero probability under every prior $P \in \mathcal{P}$. Next, we illustrate this construction method of priors for Peng's $G$-expectation ${ }^{13}$

\footnotetext{
${ }^{10}$ This topology is generated by the supremum norm $\|\omega\|_{\infty}=\sup _{t \in[0, T]}\left|\omega_{t}\right|, \omega \in \Omega$.

${ }^{11}$ Note that $P_{0}$ is not a reference measure and its technical purpose is linked to the construction of the uncertainty model. The case $P_{0} \notin \mathcal{P}$ is possible and refers to $1 \notin \mathcal{D}$ in Assumption 1.

${ }^{12}$ The set of measures is relatively compact if and only if for each sequence of closed sets $F_{n} \searrow \emptyset$ implies $\sup _{P \in \mathcal{P}} P\left(F_{n}\right) \searrow 0$. Regularity in terms of monotonic continuity of $\mathbb{E}^{\mathcal{P}}[\cdot]$ is equivalent to weak relative compactness for $\mathcal{P}$. We refer to Huber and Strassen (1973) and Denis, Hu, and Peng (2011).

${ }^{13}$ We refer to Peng (2010) for the analytic construction of $G$-expectations.
} 
Example 1 Let the uncertainty be given by a G-expectation. The volatility is associated with the volatility bounds $0<\underline{\sigma}<\bar{\sigma}$. The associated nonlinear expectation $\mathbb{E}_{G}[X]$ can be represented by $\max _{P \in \mathcal{P}} \mathrm{E}^{P}[X]=\mathbb{E}_{G}[X], \mathcal{P}$ is induced by $\mathcal{D}=\left\{a \in L^{2}(P \otimes \mathrm{d} t)\right.$ and $\mathbb{F}^{o}$-adapted $: a_{t}(\omega) \in[\underline{\sigma}, \bar{\sigma}] P_{0}$-a.s. $\}$. This is a weakly compact set of probability measure on $(\Omega, \mathcal{F}){ }^{14}$ The quadratic variation process is no longer deterministic. All the volatility uncertainty for $B$ is concentrated in the quadratic variation $\langle B\rangle$. Under every prior $P^{a}$ in $\mathcal{P}$, the volatility process is given by $\langle B\rangle_{t}^{P^{a}}=\int_{0}^{t} a_{s} \mathrm{~d} s$. This bracket process is absolutely continuous with respect to the Lebesgue measure on $[0, T]$ and its density satisfies $\underline{\sigma}^{2} t \leq\langle B\rangle_{t} \leq \bar{\sigma}^{2} t, t \in[0, T]$, $P_{0}$-a.s.

\subsection{The Commodity Space and the Price Dual}

We aim to consider contingent claims having a finite expectation for every possible prior $P \in \mathcal{P}$. In the tradition of Debreu (1959), we present an axiomatic analysis of economic equilibrium, when Assumption 1 defines the uncertainty model. We introduce the underlying space of consumption bundles $(c, C)$ consisting of consumption at time 0 and time $T$. The comprehensive set of priors prevents the consideration of a classical Lebesgue space. Nevertheless, we repeat similar steps and begin with a rather small set of reasonable random variable. Then we introduce a reasonable norm with which we accomplish the (topological) completion.

We begin to describe the state-dependent consumption good at time $T$, where we consider only claims on consumption with a finite expectation for each prior $P \in \mathcal{P}$. As in Huber and Strassen (1973), for each $\mathcal{F}$-measurable real functions $X: \Omega \rightarrow \mathbb{R}$ such that the expectation $\mathrm{E}^{P}[X]$ exists under every $P \in \mathcal{P}$, we define the upper expectation operator 15

$$
\mathbb{E}^{\mathcal{P}}[X]=\max _{P \in \mathcal{P}} \mathrm{E}^{P}[X]
$$

For a general treatment, see Denis, Hu, and Peng (2011) and the references therein. Let $\mathcal{C}_{b}(\Omega)$ denote the set of all bounded, continuous and $\mathcal{F}$-measurable real functions. The concrete description of our uncertainty model allows us to define an appropriate commodity space which considers every prior in $\mathcal{P}$ as relevant. Consequently, we suggest a norm taking every prior into account, so that we consider the capacity-type norm $c_{1, \mathcal{P}}$ on $\mathcal{C}_{b}(\Omega)$ by $c_{1, \mathcal{P}}(X)=\mathbb{E}^{\mathcal{P}}[|X|]$.

\subsubsection{The Commodity Space}

Let the closure of $\mathcal{C}_{b}(\Omega)$ under $c_{1, \mathcal{P}}$ be denoted by $\mathcal{L}^{1}(\mathcal{P})=\mathcal{L}^{1}(\Omega, \mathcal{F}, \mathcal{P}){ }^{16}$ Moreover let $L^{1}(\mathcal{P})=\mathcal{L}^{1}(\mathcal{P}) / \mathcal{N}$ be the quotient space of $\mathcal{L}^{1}(\mathcal{P})$ given by the $c_{1, \mathcal{P}}$ null elements

\footnotetext{
${ }^{14}$ See Proposition 5 in Denis and Kervarec (2013) for the weak compactness and convexity, see also Theorem 2.1.20 in Peng (2007).

${ }^{15} \mathbb{E}^{\mathcal{P}}[\cdot]$ satisfies the property of a sublinear expectation (see Peng $(2006)$ ), i.e. monotonicity, positive homogeneity, constant preserving, sub-additivity. This object builds the basis of our model.

${ }^{16}$ It is easily verified that $\mathcal{C}_{b}(\Omega) \subset \operatorname{dom}\left(\mathbb{E}^{\mathcal{P}}[\cdot]\right)=\left\{X \in L(\Omega): \mathbb{E}^{\mathcal{P}}[X]<\infty\right\}$ holds, where $L(\Omega)$ denotes the set of Borel measurable function $X: \Omega \rightarrow \mathbb{R}$.
} 
denoted by $\mathcal{N}{ }^{17}$ We do not distinguish between classes and their representatives. Two random variables $X, Y \in L^{1}(\mathcal{P})$ can be distinguished if there is a prior in $P \in \mathcal{P}$ such that $P(X \neq Y)>0 .{ }^{18}$ For the given commodity space we may introduce an order structure $X \leq Y$ if $P(X \leq Y)=1$ for every prior $P \in \mathcal{P}$. We obtain the following result 19

Proposition 1 The given triplet $\left(L^{1}(\mathcal{P}), c_{1, \mathcal{P}}(\cdot), \leq\right)$ is a Banach lattice with an $\sigma$ order continuous norm, that is $X_{n} \searrow 0$, with $X_{n} \in L^{1}(\mathcal{P})$ implies $c_{1, \mathcal{P}}\left(X_{n}\right) \searrow 0$.

As usual, we define by $L^{1}(\mathcal{P})_{+}=\left\{X \in L^{1}(\mathcal{P}): X \geq 0 \mathcal{P}\right.$-q.s. $\}$ the positive cone of $L^{1}(\mathcal{P})$. In Subsection 3.2, the fine quasi sure order structure causes a more involved notion of strict monotonicity.

\begin{tabular}{llll}
\hline Cone & Order & Monotonicity & Arbitrage \\
\hline$L^{1}(\mathcal{P})_{+}$ & $X \geq Y$ q.s. & standard & - \\
$L^{1}(\mathcal{P})_{+} \backslash\{0\}$ & $X \geq Y$ q.s. $\& X \neq Y$ & strict & weak \\
$L^{1}(\mathcal{P})_{\oplus}$ & $X \geq Y \& X \neq Y$ & semi-strict & semi weak \\
$L^{1}(\mathcal{P})_{++}$ & $X>Y$ q.s. $\forall P \in \mathcal{P}$ & weakly strict & strong \\
\hline
\end{tabular}

Table 1: Order Structures in the Commodity Space $L^{1}(\mathcal{P})$

Loosely speaking, a strictly desirable consumption bundle must be nonzero under every possible prior. In preparation, let us introduce the cone of semi-strictly positive random variables

$$
L^{1}(\mathcal{P})_{\oplus}=\left\{X \in L^{1}(\mathcal{P})_{+}: P(X>0)>0 \forall P \in \mathcal{P}\right\} .
$$

Note that this intermediate cone contains $L^{1}(\mathcal{P})_{++}=\left\{L^{1}(\mathcal{P})_{+}: X>0\right.$ q.s. $\}$, the quasi interior of $L^{1}(\mathcal{P})_{+} 20$ Accordingly, we have the following strict inclusions $L^{1}(\mathcal{P})_{++} \varsubsetneqq L^{1}(\mathcal{P})_{\oplus} \varsubsetneqq L^{1}(\mathcal{P})_{+} \backslash\{0\}$. Table 1 summarizes the different cones and their interrelation to monotonicity and possible arbitrage notions.

\subsubsection{Unambiguous Contingent Claims}

As illustrated in Figure 2, there are contingent claims which can be perfectly replicated. Such random variables are not affected by the volatility uncertainty. As we will discuss later, especially in Subsection 4.1, there is a subspace of $L^{1}(\mathcal{P})$ which becomes a natural candidate for the marketed space of perfectly replicable contingent claims, as given by

$$
\begin{aligned}
\mathrm{M}[\mathcal{P}] & =\left\{\xi \in L^{1}(\mathcal{P}): \mathbb{E}^{\mathcal{P}}[\xi]=-\mathbb{E}^{\mathcal{P}}[-\xi]\right\} \\
& =\left\{\xi \in L^{1}(\mathcal{P}): E^{P}[\xi]=E^{P^{\prime}}[\xi] \text { for all } P, P^{\prime} \in \mathcal{P}\right\} .
\end{aligned}
$$

\footnotetext{
${ }^{17}$ One can show that these null elements are $\mathcal{P}$-quasi surely zero.

${ }^{18}$ In a setting with equivalent priors, i.e. priors sharing the same sets of mass zero, this implies that $P(X \neq Y)>0$ for all $P \in \mathcal{P}$.

${ }^{19}$ This is important for the application of an abstract existence result for quasi equilibria. However, we take a different approach to prove existence, see also Remark 2.

${ }^{20}$ By Proposition $1, L^{1}(\mathcal{P})$ is a Banach lattice. The representation follows then by Lemma 4.15 in Abramovich and Aliprantis (2002).
} 
Random variables in $\mathrm{M}[\mathcal{P}]$ are called $\mathcal{P}$-unambiguous. For another set of priors $\mathcal{Q}$, the notion of $\mathcal{Q}$-unambiguity (in $L^{1}(\mathcal{P})$ ) is still meaningful and well-defined. Mean unambiguity is strongly related to ambiguity neutrality. For instance, we may take the viewpoint of Epstein and Zhang (2001) and consider the Dynkin system of unambiguous events $\mathcal{U}(\mathcal{P})=\{A \in \mathcal{F}: \bar{P}(A)$ is constant for all $P \in \mathcal{P}\}$.

\subsubsection{The Price Space}

We turn now to the space of price systems on $\mathbb{R} \times L^{1}(\mathcal{P})$. A model which aims to observe the existence of a general equilibrium should first of all clarify what price system decentralizes an allocation. As it is common, we suppose a linear price system $\Psi: \mathbb{R} \times L^{1}(\mathcal{P}) \rightarrow \mathbb{R}$. Moreover we require continuity under the topology of the $c_{1, \mathcal{P}}$-norm ${ }^{21}$ We discuss the topological dual of $L^{1}(\mathcal{P})$. For our purposes we need to determine market prices via marginal rates of the agents. For the existence proof for equilibrium, the following result is of importance.

Proposition 2 Elements in the topological dual of $\left(L^{1}(\mathcal{P}), c_{1, \mathcal{P}}\right)$ can be represented by an absolutely continuous measure:

$$
L^{1}(\mathcal{P})^{*} \supset\left\{l(\cdot)=\int \cdot \mathrm{d} \mu=\mathrm{E}^{P}[\psi \cdot]: P \in \mathcal{P} \text { and } \psi \in L^{\infty}(P)\right\}=\tilde{L}^{1}(\mathcal{P})^{*}
$$

The subspace $\tilde{L}^{1}(\mathcal{P})$ in Proposition 2 is smaller than $L^{1}(\mathcal{P})$. Same arguments, as in Proposition 1 , show that $\left(\tilde{L}^{1}(\mathcal{P}), c_{1, \mathcal{P}}(\cdot), \leq\right)$ is an order continuous Banach lattice 22

Remark 1 In Lemma 1 we consider a class of utility functionals on $L^{1}(\mathcal{P})$ such that there are super-gradients even in $\tilde{L}^{1}(\mathcal{P})^{*}$. In principle, the dual of $\tilde{L}^{1}(\mathcal{P})$ seems to be more acceptable. On the other side, it is unclear how to work within $\tilde{L}^{1}(\mathcal{P})$, when we apply results from the dynamic theory of $G$-expectation, whose natural domain is $L^{1}(\mathcal{P})$. Moreover, some convergence results are only available for $L^{1}(\mathcal{P})$ (see the beginning of the Appendix).

The representation in Proposition 2 has similarities to the duality of Lebesgue spaces from classical measure theory, when only one prior $P$ describes the uncertainty. Note that the stronger capacity norm $c_{1, \mathcal{P}}(\cdot)$ in comparison to the single prior $L^{1}(P)$-norm implies a richer dual space, controlled by the set of priors $\mathcal{P}{ }^{23}$ Let us introduce the space of semi-strictly positive functionals

$$
L^{1}(\mathcal{P})_{\oplus}^{*}=\left\{l \in L^{1}(\mathcal{P})^{*}: l(\cdot)=\mathrm{E}^{P}[\psi \cdot] \text { with } P \in \mathcal{P} \text { and } \psi \in L^{\infty}(P)_{++}\right\},
$$

where $L^{\infty}(P)_{++}$denotes the cone of $P$-a.s. strictly positive and bounded random variables. Suppose $l \in L^{1}(\mathcal{P})_{\oplus}^{*}$, then $l$ may not be strictly positive, i.e. $l(Y)=0$

\footnotetext{
${ }^{21}$ Later on we assume semi-strict monotonicity of preferences. This guarantees semi-strictly positive prices. Since $\mathbb{R} \times L^{1}(\mathcal{P})$ is a Banach lattice, this implies norm continuity.

${ }^{22}$ For more details, we refer to Section 2, Lemma 4.1 and Proposition 4.1 in Bion-Nadal and Kervarec (2012).

${ }^{25}$ With the explicit representation in Proposition 2, the weak topology of the dual pairing is tractable and allows us to apply standard convergence results from measure theory.
} 
if $Y \in L^{1}(\mathcal{P})_{+} \backslash\{0\}{ }^{24}$ This indicates that we need a weaker notion than strict positivity. In Table 2, we give the different dual cones and their interrelation to the representation property. Similarly to the commodity space we have different order structures with respect to its order dual. Specifically, we compare the representing measure of Proposition 2. Furthermore,

\begin{tabular}{llll}
\hline Dual Cone & Order & Positivity & Repr. d $\mu=\psi \mathrm{d} P$ of $l$ \\
\hline$L^{1}(\mathcal{P})_{+}^{*}$ & $l \geq 0$ on $L^{1}(\mathcal{P})_{+}$ & standard & $\psi \in L^{\infty}(P)_{+} \& P \in \mathcal{P}$ \\
$\tilde{L}^{1}(\mathcal{P})_{++}^{*}\{0\}$ & $l>0$ on $\tilde{L}^{1}(\mathcal{P})_{+}+\{0\}$ & strict & $\psi \in L^{\infty}(P)_{++} \& P \in \mathcal{P}$ din $^{25}$ \\
$L^{1}(\mathcal{P})^{*}$ & $l>0$ on $L^{1}(\mathcal{P})_{\oplus}$ & semi-strict & $\psi \in L^{\infty}(P)_{++} \& P \in \mathcal{P}$ \\
$L^{1}(\mathcal{P})_{++}^{\oplus}$ & $l>0$ on $L^{1}(\mathcal{P})_{++}$ & weakly strict & $\psi \in L^{\infty}(P)_{+} \backslash\{0\}$ \\
\hline
\end{tabular}

Table 2: Order Structures in the Dual of $L^{1}(\mathcal{P})$

the following result shows that exactly semi-strictly positive random variables have strictly positive values with respect to functionals in $L^{1}(\mathcal{P})_{\oplus}^{*}$.

Corollary 1 Let $l: L^{1}(\mathcal{P}) \rightarrow \mathbb{R}$ be a linear and continuous functional, we have: $l \in L^{1}(\mathcal{P})_{\oplus}^{*} \Leftrightarrow l(X)>0$ for all $X \in L^{1}(\mathcal{P})_{\oplus}$.

Now, the representing measure $\mu$ of a linear and $c_{1, \mathcal{P}}$-continuous functional can be decomposed by $P$ and $\psi$. The $P$-almost surely strictly positive random variable $\psi \in$ $L^{\infty}(P)_{++}$can be seen as a state price density under the cohesive probability model $P$. This allows us to represent every semi-strictly positive, normalized, continuous and linear price system on $\mathbb{R} \times L^{1}(\mathcal{P})$ as

$$
\Psi(x, X)=\pi(x)+\Pi(X)=\pi \cdot x+\mathrm{E}^{Q}[X]
$$

where $\pi>0$. The equilibrium price measure given by $Q(A)=\int_{A} \psi(\omega) \mathrm{d} P(\omega)$, $A \in \mathcal{F}$, describes the value of any claim in terms of an expected payoff, where $\psi$ is normalized to unit expectation under $P \in \mathcal{P}$. To sum up, our commodity-price pair is given by $\left(\mathbb{R} \times L^{1}(\mathcal{P}), \mathbb{R} \times L^{1}(\mathcal{P})^{*}\right)$.

\subsection{Variational Preferences}

A priori, each agent is faced with the same objective uncertainty model $(\Omega, \mathcal{F}, \mathcal{P})$. In this situation the heterogeneity is induced by different ambiguity attitudes which we describe below.

Every agent is determined by an initial endowment $(e, E) \in \mathbb{R}_{+} \times L^{1}(\mathcal{P})_{+}$, and a utility functional $V$ on $\mathbb{R}_{+} \times L^{1}(\mathcal{P})_{+}$being additively separable, so that we can write $V(x, X)=u^{0}(x)+U(X)$. We describe the utility functional $U$ on $L^{1}(\mathcal{P})_{+}$for an

\footnotetext{
${ }^{24}$ This can be seen as follows. Let $\hat{P}(Y>0)>0$ and $Y=0 P$-a.s. for every $P \in \mathcal{P} \backslash\{\hat{P}\}$ and let $X \mapsto l(X)=\mathrm{E}^{P_{l}}[\psi X]$ such that $P_{l}, \hat{P}$ are mutually singular and $\psi>0 P^{l}$-a.s., hence $l(Y)=0$.

${ }^{25}$ Note that $\mathcal{P}_{\text {can }}$ denotes the canonical equivalence class, which we mention in Example 3 of Subsection 3.2, below. For details we refer to section 4 in Bion-Nadal and Kervarec (2012) and especially Definition 4.3 therein.
} 
arbitrary agent, where the positive cone is induced by the order relation of Section 3.1. As we consider preference relations $\succeq$ on $L^{1}(\mathcal{P})_{+}$, convexity and continuity can be defined in a standard way. Monotone preferences are directly related to the order structure and the set of priors $\mathcal{P}$. We state a weak notion of strict monotonicity on $L^{1}(\mathcal{P})_{+} \cdot{ }^{26}$

Definition 1 A preference relation is called semi-strictly monotone at $X \in$ $L^{1}(\mathcal{P})_{+}$, if $X+Z \succ X$ for all $Z \in L^{1}(\mathcal{P})_{\oplus}$.

In Remark 1 below, we discuss in more detail why this modified strict monotonicity condition is more appropriate when mutually singular priors constitute the uncertainty. We allow for variational preferences introduced and axiomatized by Maccheroni, Marinacci, and Rustichini (2006):27

$$
C \succeq X \Longleftrightarrow \min _{P \in \mathcal{P}}\left(\mathrm{E}^{P}[u(C)]+\mathfrak{c}(P)\right) \geq \min _{P \in \mathcal{P}}\left(\mathrm{E}^{P}[u(X)]+\mathfrak{c}(P)\right),
$$

where $u: \mathbb{R}_{+} \rightarrow \mathbb{R}$ is a utility index and $\mathfrak{c}: \mathcal{P}_{S} \rightarrow \mathbb{R}$ is an ambiguity index. For each $C \in L^{1}(\mathcal{P})_{+}$define the set of effective probability scenarios

$$
M(C)=\underset{P^{\prime} \in \mathcal{P}}{\operatorname{argmin}} \mathrm{E}^{P^{\prime}}[u(C)]+\mathfrak{c}\left(P^{\prime}\right) .
$$

As we show in Lemma 1, this set is proportional to the superdifferential of the utility. Such priors minimize the variational utility at $C$. We assume that variational preferences are defined on a weakly closed set of priors for the uncertainty model $\mathcal{P}$, i.e. $\operatorname{dom}(\mathfrak{c})=\left\{P \in \mathcal{P}_{S}: \mathfrak{c}(P)<\infty\right\} \subseteq \mathcal{P}$. The case $\operatorname{dom}(\mathfrak{c})=\{P\}$ corresponds to an expected utility under $P \in \mathcal{P}$. The following lemma gives standard properties of the variational utility functional.

Lemma 1 Let $u: \mathbb{R}_{+} \rightarrow \mathbb{R}$ be monotone, strictly concave, continuous and differentiable on $\mathbb{R}_{++}$. Let $\mathfrak{c}: \mathcal{P}_{S} \rightarrow[0, \infty]$ be grounded ${ }^{28}$, convex, and weakly lower semi-continuous. Define the utility functional $U: L^{1}(\mathcal{P})_{+} \rightarrow \mathbb{R}$ by (1).

Then we have, $U: L^{1}(\mathcal{P})_{+} \rightarrow \mathbb{R}$ is

1. monotone and semi-strictly monotone if $u$ is strictly monotone.

2. concave, not strictly concave on $L^{1}(\mathcal{P})_{+}$and strictly concave on $\mathrm{M}[\mathcal{P}]$.

3. $c_{1, \mathcal{P}}$-upper semi-continuous. If the penalty term is linear on $\operatorname{dom}(\mathfrak{c})$, then

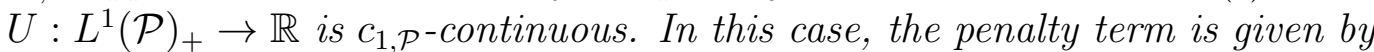
$\mathfrak{c}(P)={ }_{L^{1}(\mathcal{P})}\langle\phi, P\rangle_{L^{1}(\mathcal{P})^{*}}=\mathrm{E}^{P}[\phi]$, for some $\phi \in L^{1}(\mathcal{P})$.

4. The superdifferential of $U: L^{1}(\mathcal{P})_{+} \rightarrow \mathbb{R}$ at $C$ is given by $\partial U(C)=$ $\left\{\mu: \mathcal{F} \rightarrow \mathbb{R}: \mathrm{d} \mu=u^{\prime}(C) \mathrm{d} P, P \in M(C)\right\}$

\footnotetext{
${ }^{26}$ An alternative notion of weak strict monotonicity could refer to a cone $L^{1}(\mathcal{P})_{X}=\{Y \in$ $\left.L^{1}(\mathcal{P})_{+}: P(Y>0)>0 \forall P \in M(X)\right\}$ depending on the effective priors $M(X)$ at $X \in L^{1}(\mathcal{P})_{+}$, defined below. In this situation strict utility improving consumption at $X$ refers to the intermediate cone $L^{1}(\mathcal{P})_{X}$, heavily depending on $X$. Another alternative is the local monotonicity concept in Nutz and Soner (2012).

${ }^{27}$ In their setting the domain of the preference relation is the space of simple acts.

${ }^{28}$ This means that its infimum value is zero.
} 
The lemma is an extension to the case of infinite (uncountable) states, see Rigotti and Shannon (2012) for the finite state case. This indicates that the present commodity price duality is tractable. For finite valued measurable functions, the explicit formula of the superdifferential is proven in Maccheroni, Marinacci, and Rustichini (2006). In the following we present examples to illustrate the usefulness and flexibility of variational preferences. The first example refers to the classical maxmin preferences found in Gilboa and Schmeidler (1989). The second example refers to anchored preferences axiomatized by Faro (2009).

Example 2 1. Maxmin Preferences: An agent with maxmin preferences is modeled by the following criterion

$$
U(C)=-\mathbb{E}^{\mathcal{P}}[-u(C)]=\min _{P \in \mathcal{P}} \mathrm{E}^{P}[u(C)]+\delta_{\mathcal{P}}(P),
$$

where $\mathfrak{c}=\delta_{\mathcal{P}}: \mathcal{P}_{S} \rightarrow[0, \infty]$ is the convex indicator function of $\operatorname{dom}(\mathfrak{c})=\mathcal{P}$.

2. Anchored maxmin preferences: Fix an initial endowment $E \in L^{1}(\mathcal{P})_{+}$. We can define the following anchored preference representation

$$
U(C)=\min _{P \in \operatorname{dom}(\mathfrak{c})} \mathrm{E}^{P}[u(C)-u(E)], \quad \mathfrak{c}(P)= \begin{cases}\mathrm{E}^{P}[u(E)] & \text { if } P \in \operatorname{dom}(\mathfrak{c}) \\ +\infty, & \text { otherwise }\end{cases}
$$

Here the penalty term is linear, with $\phi=-u(E){ }^{29}$

In multiple prior models based on a reference measure, i.e. all other priors are absolutely continuous, it is possible to consider the relative entropy in terms of $\mathfrak{c}$. The usage is limited and does not apply to mutually singular priors directly, as the following example illustrates.

Example $3 \lambda$-Relative Entropy: In this example, we concentrate on the cone $\tilde{L}^{1}(\mathcal{P})_{+}$. In case of a penalty term associated to the relative entropy we need some preparation for the construction. We introduce some synthetic probability measure. As discussed in Bion-Nadal and Kervarec (2012), there is a canonical equivalence class $R\left(c_{1}, \mathcal{P}\right)=\mathcal{P}^{\text {can }}$ of probability measures. This class is based on a countable dense subset $\left\{P^{n}\right\}_{n \in \mathbb{N}}$ of $\mathcal{P}$. Taking a sequence of real number $\left\{\lambda_{n}\right\}_{n \in \mathbb{N}}$ with $\lambda_{n}>0$ such that $\sum \lambda_{n}=1$ the resulting probability measure $P^{\lambda}=\sum \lambda_{n} P^{n}$ is in $\mathcal{P}^{\text {can }}$. Let a reference measure $P_{\lambda}$ be fixed. The $\lambda$-relative entropy $R^{\lambda}(\cdot \| \mathcal{P}): \mathcal{P}_{S} \rightarrow[0, \infty]$ is defined by $R^{\lambda}(P \| \mathcal{P})=\int_{\Omega} \log \left(\frac{\mathrm{d} P}{\mathrm{~d} P_{\lambda}}\right) \mathrm{d} P_{\lambda}$. Priors closer to the dominating prior $P_{\lambda}$ have a larger influence on the utility evaluation. Finally we can write the utility functional as

$$
U_{\left\lceil\tilde{L}^{1}(\mathcal{P})_{+}\right.}(C)=\min _{P \in \mathcal{P}} \mathrm{E}^{P}[u(C)]+\mathfrak{c}(P)=\min _{P \in \mathcal{P}} \mathrm{E}^{P}[u(C)]+\theta R^{\lambda}(P \| \mathcal{P}),
$$

where $\theta \in \mathbb{R}$ is an intensity parameter. Note that this relative entropy formulation heavily depends on the parameter $\lambda$ for the synthetic measure $P^{\lambda}$.

${ }^{29}$ In Dana and Riedel (2013) such anchored preferences are related to Bewley preferences, see Bewley (2002). However, in their discrete time framework the uncertainty is modeled by a set of mutually equivalent priors. 
We close the section with a discussion on semi-strict monotonicity.

Remark 2 1. Strict monotonicity is usually defined by $U(X+Y)>U(X)$ for some $Y \in L^{1}(\mathcal{P})_{+} \backslash\{0\}$. The singularities of the priors do not allow for such a notion of monotonicity. We illustrate this issue for maxmin preferences in Example 2.1. Let $Y \in L^{1}(\mathcal{P})_{+}$has only one effective prior $P^{Y} \in \mathcal{P}$. Then we may have

$$
U(Y)=\min _{P \in \mathcal{P}} \mathrm{E}^{P}[u(Y)]=\mathrm{E}^{P}[u(Y)]=\mathrm{E}^{P}[u(0)]=U(0) \text {, where } P \neq P^{Y} .
$$

This means, the commodity bundle $Y$ may not be strictly desirable in comparison to zero consumption. From this point of view variational preferences seem to be rather consistent with semi-strictly preferences. Another argument refers to the representation property of the topological dual. As pointed out in Lemma 1, the utility gradients can be decomposed, with the strict positivity of the density part satisfied for semi-strict monotone and concave variational preferences (see also Corollary 1). In this situation, semi-strict positive functionals are compatible with the utility gradients. The equilibrium results in Section 4 are strongly connected to this issue.

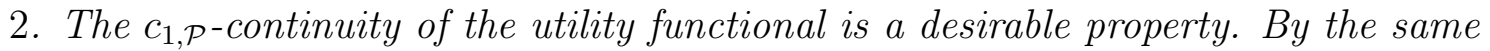
argument as for the classical Lebesgue space, related to some probability space, we have an empty interior of the positive cone $L^{1}(\mathcal{P})_{+}$. Monotone and concave variational utility defined on the whole space are $c_{1, \mathcal{P}}$-continuous. This follows for instance from an application of the extended Namioka-Klee Theorem in Biagini and Frittelli (2010).

\section{Equilibria and Implementation}

This Section is divided into three parts. In a preliminary step we introduce the martingale theory of the considered conditional sublinear expectation. Then we establish the existence of an equilibrium allocation and discuss some new structural properties. In the last step we achieve the implementation of the equilibrium allocation into a Radner economy.

\subsection{A Detour: Spanning and Martingales}

In order to establish a Radner implementation, we introduce a new sublinear expectation, generated via the supremum of the adjusted priors. Due to the present uncertainty model $\mathcal{P}$, a new well-behaved conditioning principle is needed. We roll out the dynamics of security markets by introducing the concept of conditional sublinear expectations. The involved implementation via a security market accounts for such well-behaved nonlinear expectations.

We proceed similarly to the single prior case, where the Radner implementation in continuous time is based on a certain classical martingale representation property. In the present situation, the multiple prior model enforces a conditional sublinear expectation which spawns an elaborated martingale representation theorem. As we indicate at the end of this subsection an effect on the space of unambiguous claims is apparent. A possible replication of the claim via the security market provokes 
the appearance of incomplete markets.

\section{Structure of Priors}

For the purpose of a martingale representation theorem under a conditional sublinear expectation we need the following notion of stability under pasting for $\mathcal{P}$, also called fork-convexity. In essence, this property refers to a rational updating principle. Before, we define the set of priors with a time-depending restriction on the related sub $\sigma$-field

$$
\mathcal{P}(t, P)^{o}=\left\{P^{\prime} \in \mathcal{P}: P=P^{\prime} \text { on } \mathcal{F}_{t}^{o}\right\} \text {, with } t \in[0, T] \text { and } P \in \mathcal{P} \text {. }
$$

This set of priors consists of all extensions of $P: \mathcal{F}_{t}^{o} \rightarrow[0,1]$ from $\mathcal{F}_{t}^{o}$ to $\mathcal{F}$ in $\mathcal{P}$. More precisely, this is the set of all probability measures in $\mathcal{P}$ defined on $\mathcal{F}$ that agree with $P$ in the events up to time $t$.

Assumption 2 The set of priors $\mathcal{P}$ is stable under pasting, i.e. for every $P \in \mathcal{P}$, every $\mathbb{F}^{o}$-stopping time $\tau, B \in \mathcal{F}_{\tau}^{o}$ and $P_{1}, P_{2} \in \mathcal{P}\left(\mathcal{F}_{\tau}^{o}, P\right)$, the set of priors $\mathcal{P}$ contains again the prior $P_{\tau}$ given by

$$
P_{\tau}(A)=\mathrm{E}^{P}\left[P_{1}\left(A \mid \mathcal{F}_{\tau}^{o}\right) 1_{B}+P_{2}\left(A \mid \mathcal{F}_{\tau}^{o}\right) 1_{B^{c}}\right], \quad A \in \mathcal{F}_{\tau}^{o}
$$

Note, that we use the raw filtration $\mathbb{F}^{o}$. The stability under pasting property is closely related to dynamic consistency or rectangularity of Epstein and Schneider (2003). However in the present volatility uncertainty setting these notions are not equivalent. ${ }^{30}$ For details we refer to Section 3 in Nutz and Soner (2012). For instance, the set of priors which defines a $G$-expectation, illustrated in Example 1, satisfies automatically Assumption 2.

\section{Information Structure}

The usual conditions of a rich $\sigma$-field at time 0 is widely used in Mathematical Finance 31 But the economic meaning is questionable. In our uncertainty model of mutually singular priors we can augment, similarly to the classical case, the right continuous filtration given by $\mathbb{F}^{+}=\left\{\mathcal{F}_{t}^{+}\right\}_{t \in[0, T]}$ where $\mathcal{F}_{t}^{+}=\bigcap_{s>t} \mathcal{F}_{t}^{o}$ for $t \in[0, T)$. The second step is to augment the minimal right continuous filtration $\mathbb{F}^{+}$by all polar sets of $\left(\mathcal{P}, \mathcal{F}_{T}^{o}\right)$, i.e. $\mathcal{F}_{t}=\mathcal{F}_{t}^{+} \vee \mathcal{N}\left(\mathcal{P}, \mathcal{F}_{T}^{o}\right)$, see Appendix A.1 for details. This augmentation is strictly smaller than the universal enlargement procedure ${ }^{32}$ Note that the augmentation does not affect the commodity space of equivalence classes, whose elements are $\mathcal{P}$-q.s. indistinguishable. Additionally we have $\mathcal{B}(\Omega)=\mathcal{F}_{T}^{o}$ and $\mathcal{F}_{T}^{o}=\mathcal{F}_{T} \mathcal{P}$-q.s.

This choice is economically reasonable, because the initial $\sigma$-field does not contain all 0-1 limit events, see Section 4.1 in Nutz and Soner (2012). In nearly all continuous-time Finance models, such a rich initial $\sigma$-field is assumed. This implies a rich knowledge of every decision maker about events in the long run. In Huang (1985) one can find a detailed discussion of information structures for asset prices

\footnotetext{
${ }^{30}$ Lemma 8 in Appendix B of Riedel (2009) shows the equivalence of these concepts, when the priors are mutually equivalent.

${ }^{31}$ One reason may be, that in this case the full stochastic calculus is applicable.

${ }^{32}$ This means $\mathcal{F}_{t} \varsubsetneqq \bigcap_{P \in \mathcal{P}} \sigma\left(\mathcal{F}^{+}, \mathcal{N}\left(P, \mathcal{F}_{t}^{o}\right)\right)$, for $t \in[0, T]$.
} 
and trading strategies, when the uncertainty is given by a probability space.

\section{Conditional Sublinear Expectation}

We introduce the dynamics and the different notions of martingales of our underlying uncertainty model $(\Omega, \mathcal{F}, \mathcal{P})$. The so called strong formulation of uncertainty in Assumption 1 guarantees the existence of a martingale concept which allow for a martingale representation. The efficient use of information is often formalized by the concept of conditional expectation. Implicitly, this depends on the uncertainty structure and the given filtration. Due to the pasting property of $\mathcal{P}$ we have a universal conditional expectation being under every prior almost surely equal to the essential supremum of relevant conditional expectations. This concept is formulated in the following definition.

Definition 2 A set of priors $\mathcal{P}$ has the aggregation property in $L^{1}(\mathcal{P})$ if for all $X \in L^{1}(\mathcal{P})$ and $t \in[0, T]$, there exists an $\mathcal{F}_{t}$-measurable random variable $\mathbb{E}_{t}^{\mathcal{P}}[X] \in$ $L^{1}(\mathcal{P})$ such that

$$
\mathbb{E}_{t}^{\mathcal{P}}[X]=\operatorname{P}_{P^{\prime} \in \mathcal{P}(t, P)}^{\operatorname{ess} \sup } \mathrm{E}^{P^{\prime}}\left[X \mid \mathcal{F}_{t}\right] \quad P \text {-a.s. for all } P \in \mathcal{P} .
$$

Note that in the definition the random variable is defined in the quasi sure sense. The linear conditional expectation under a probability space has strong connections to a positive linear projection operator. In the presence of multiple priors, the conditional updating in an ambiguous environment involves a sublinear projection $\mathbb{E}_{t}^{\mathcal{P}}: L^{1}(\mathcal{P}) \rightarrow L_{t}^{1}(\mathcal{P})$, where $L_{t}^{1}(\mathcal{P}) \subset L^{1}(\mathcal{P})$ denotes the closed subspace of $\mathcal{F}_{t}$ measurable random variables. In this regard the aggregation property just states that we can find a well-defined sequence of conditional expectations satisfying a rational updating principle. The weak compactness and stability under pasting allows for such a conditional sublinear expectation.

Lemma 2 Under Assumption 1 and $2, \mathcal{P}$ satisfies the aggregation property. Moreover, we have $\mathbb{E}_{s}^{\mathcal{P}} \circ \mathbb{E}_{t}^{\mathcal{P}}=\mathbb{E}_{s}^{\mathcal{P}}, s \leq t$.

Without a well-behaved conditional expectation, the introduction of a martingale or its representation seems unreproducible ${ }^{33}$ Now, we introduce martingales under the conditional expectation $\mathbb{E}_{t}^{\mathcal{P}}$. Fix a random variable $X \in L^{1}(\mathcal{P})$. The sublinearity of the dynamic conditional expectation defines a martingale similarly to the single prior setting ${ }^{34}$ as being its own estimator.

Definition 3 An $\mathbb{F}$-adapted process $\left(X_{t}\right)_{t \in[0, T]}$ is a $\mathcal{P}$-martingale if

$$
X_{s}=\mathbb{E}_{s}^{\mathcal{P}}\left[X_{t}\right] \quad \mathcal{P} \text {-q.s. for all } s \leq t .
$$

We call $X$ a symmetric $\mathcal{P}$-martingale if $X$ and $-X$ are both $\mathcal{P}$-martingales.

\footnotetext{
${ }^{33}$ Without the weak compactness of $\mathcal{P}$, a construction of random variables in the quasi sure sense involves more technical difficulties. However, in this situation one can take the separability condition of Soner, Touzi, and Zhang (2012b), see also Example 4.14 therein. An aggregation result, in the sense of Definition 2, can then be observed with the so called Hahn property of Cohen (2011). Here the definition of an ess sup in the quasi sure sense approaches the aggregation property.

${ }^{34}$ For the multiple prior case with equivalent priors we refer to Riedel (2009).
} 
The nonlinearity of the conditional expectation implies that if a process $\left(X_{t}\right)$ is a martingale under $\left(\mathbb{E}_{t}^{\mathcal{P}}\right)_{t \in[0, T]}$, then $-X$ is not necessarily a martingale ${ }^{35}$ As we will discuss in detail, a fair game refers to the symmetric martingale property. In this case, the process is equivalently a $P$-martingale under every $P \in \mathcal{P}$. In subsection 4.3 we discuss the relationship to asset prices under the sublinear expectation generated by $\mathcal{P}$.

In a dynamic trading setting, it is essential if a contingent claim $X \in L^{1}(\mathcal{P})$ can be represented in terms of a stochastic integral. As mentioned in the Introduction this corresponds to the mean unambiguity property, introduced in Section 3.1. For the replication of a claim, the following result is central. It can be seen as a generalized martingale representation theorem, when the uncertainty is given by the present mutually singular uncertainty model, see Nutz and Soner (2012) for a proof.

Martingale Representation: Under Assumption 1 and 2, we have for every $X \in L^{1}(\mathcal{P})$ a unique pair $(\theta, K)^{36}$ of $\mathbb{F}$-predictable processes with

1. $\theta$ such that $\int_{0}^{T}\left|\theta_{s}\right|^{2} \mathrm{~d}\langle B\rangle_{s}<\infty \mathcal{P}$-q.s.,

2. $K$ such that all paths of $\left(K_{t}\right)$ are càdlàg, nondecreasing and $K_{T} \in L^{1}(\mathcal{P})$,

$$
\text { such that } \mathbb{E}_{t}^{\mathcal{P}}[X]=\mathbb{E}_{0}^{\mathcal{P}}[X]+\int_{0}^{t} \theta_{s} \mathrm{~d} B_{s}-K_{t} \quad \text { for all } t \in[0, T], \quad \mathcal{P} \text {-q.s. }
$$

The positive and increasing process $K$ in the representation is new and can be understood as a correction term. The sublinear conditional expectation allows for biased martingales, i.e. we only have $\mathrm{E}^{P}\left[-K_{T}^{X}\right]=0$ if and only if $P \in \operatorname{argmax}_{P \in \mathcal{P}} \mathrm{E}^{P}[X]$. Here, $K^{X}$ is the output of the martingale representation theorem applied with respect to $X \in L^{1}(\mathcal{P})$.

Remark 3 1. Already at this stage, the interplay between the existence and the structure of a competitive equilibrium and absence of arbitrage opportunities are at work. As illustrated in Vorbrink (2010) in the G-framework (see Example 1) absence of weak arbitrage (see Table 1 ) does not imply $\mathrm{E}^{P}\left[-K_{T}\right]=0$ for every $P \in \mathcal{P}$. Note that this arbitrage notion is consistent with strictly monotone preferences, stated in Table 1 and refers to a robust approach to finance.

If an exchange economy is in equilibrium, net trades should not admit for arbitrage. But, by Proposition 2 the equilibrium price system perceives only $P^{*}$-a.s. events, since the representing measure $\mu$ of the equilibrium price system can be decomposed by $\mathrm{d} \mu=\psi \mathrm{d} P^{*}$. The value of net trades $\xi \in L^{1}(\mathcal{P})$ should not differ under such equilibrium priors $P^{*}$. Therefore, the case $P^{\prime}\left(K_{T}^{\xi} \neq 0\right)>0$ must refer to a nonequilibrium prior $P^{\prime}$, see Example 5 for an application of this issue.

2. In the $G$-framework the compensation part can be written more explicit, when $X$

\footnotetext{
${ }^{35}$ Representations of non symmetric martingales can be formulated via a so called second order backward stochastic differential equation (2BSDE). This concept is introduced in Cheridito, Soner, Touzi, and Victoir (2007) and developed further in Soner, Touzi, and Zhang (2012a).

${ }^{30}$ The pair is unique up to $\{\mathrm{d} s \times P, P \in \mathcal{P}\}$-polar sets. More precisely, the process $K$ is an aggregated object under the Continuum Hypothesis, see Remark 4.17 of Nutz and Soner (2012) and paragraph 8 and 9 of Chapter 0 in Dellacherie and Meyer (1978).
} 
is contained in a (uncertain) subset of $L^{1}(\mathcal{P})$ :

$$
K_{t}=\int_{0}^{t} \eta_{r} \mathrm{~d}\left\langle B^{G}\right\rangle_{r}-\int_{0}^{t} G\left(\eta_{r}\right) \mathrm{d} r, \quad t \in[0, T],
$$

where $B^{G}$ is the so called $G$-Brownian motion ${ }^{37}$ and $\eta$ is an endogenous output of the martingale representation, so that $K$ is now a function of $\eta$. Example 5 and 6 make use of this fact.

As such it is an open problem, if every $X \in L^{1}(\mathcal{P})$ can be represented in this complete form. We refer to Peng, Song, and Zhang (2013) for the latest discussion, on the complete representation property.

The following corollary gives an alternative representation and a justification of unambiguous random variables. It illustrates which random variables have the replication property in terms of a stochastic integral. The space of feasible integrands $\Theta(B)$ is given below in Subsection 4.3.

Corollary 2 The marketed space $\mathrm{M}[\mathcal{P}]$ of unambiguous contingent claims is a $c_{1, \mathcal{P}^{-}}$ closed subspace of $L^{1}(\mathcal{P})$. More precisely, we have

$$
\mathrm{M}[\mathcal{P}]=\left\{\xi \in L^{1}(\mathcal{P}): \xi=\mathbb{E}^{\mathcal{P}}[\xi]+\int_{0}^{T} \theta_{s} \mathrm{~d} B_{s} \text { for some } \theta \in \Theta(B)\right\} .
$$

Furthermore, the stochastic integral has continuous paths $\mathcal{P}$-q.s.

The notion of perfect replication is associated to the situation when $K \equiv 0$. Exactly at this step the martingale representation comes into play. This space of random variables is strongly related to symmetric martingales. More precisely, elements in $\mathrm{M}[\mathcal{P}]$ generate symmetric martingales, via the successive application of the conditional sublinear expectation along the augmented filtration $\mathbb{F}$. In the lights of Corollary 2, the analogy between unambiguous events and unambiguous random variables becomes apparent 38 Note that this analogy is already used and indicated in Beißner (2012), where a notion of ambiguity and risk neutral valuation is considered.

\subsection{Existence of Arrow-Debreu Equilibrium}

With the discussion of the primitives in Section 3, we introduce now the heterogeneous agent economy, consisting of a finite set of individuals $\mathbb{I}=\{1, \ldots, I\}$ consuming at time $t=0$ and $t=T$. The economy is given by $\mathcal{E}(e, E)=$ $\left(\mathbb{R}_{+} \times L^{1}(\mathcal{P})_{+},\left\{V_{i},\left(e_{i}, E_{i}\right)\right\}_{i \in \mathbb{I}}\right)$, where the initial endowment of agent $i$ satisfies $\left(e_{i}, E_{i}\right) \in \mathbb{R}_{+} \times L^{1}(\mathcal{P})_{+}$. Her utility is given by $V_{i}: \mathbb{R}_{+} \times L^{1}(\mathcal{P})_{+} \rightarrow \mathbb{R}$ such that $(c, C) \mapsto u_{i}^{0}(c)+U_{i}(C)$. The utility $U_{i}: L^{1}(\mathcal{P})_{+} \rightarrow \mathbb{R}$ is a variational functional,

\footnotetext{
${ }^{37}$ As already mentioned in Example 1, a $G$ expectation can be induced by some volatility bounds. Here, the function $G$ is given by $\eta \mapsto G(\eta)=\frac{1}{2} \sup _{\sigma \in[\sigma, \bar{\sigma}]} \sigma \cdot|\eta|$.

${ }^{38}$ Under one prior or a set of mutually equivalent priors, indicator functions are elements of the related Lebesgue space. In our setting this is not necessarily true, since $\omega \mapsto 1_{A}(\omega)$, for some $A \in \mathcal{F}$ is not continuous.
} 
introduced in Section 3.2, with utility functions $u_{i}^{0}, u_{i}^{T}: \mathbb{R}_{+} \rightarrow \mathbb{R}$ and ambiguity indexes $\mathfrak{c}_{i}, 39$ we have

$$
V_{i}(c, C)=u_{i}^{0}(c)+\min _{P \in \mathcal{P}} \mathrm{E}^{P}\left[u_{i}^{T}(C)\right]+\mathfrak{c}_{i}(P) .
$$

The aggregate endowment of the economy is denoted by $(e, E) \in \mathbb{R}_{+} \times L^{1}(\mathcal{P})_{+}$. Note that we allow for a heterogeneity in the sets of priors. This can be achieved via different domains of the penalty terms $\mathfrak{c}_{i}$, see also the last part of Assumption 3.

\subsubsection{Efficient Allocations and Sharing Rules}

We describe the optimal allocation of resources by the following problem. A weighting $\alpha \in \Delta_{I}$, where $\Delta_{I}=\left\{\alpha \in \mathbb{R}_{+}^{I}: \sum \alpha_{i}=1\right\}$ denotes the $I$-dimensional simplex, induces a representative utility $V_{\alpha}(c, C):=\sum \alpha_{i} V_{i}\left(c_{i}, C_{i}\right)$.

An allocation $(\bar{c}, \bar{C})=\left(\left(c_{1}, C_{1}\right) \ldots,\left(c_{I}, C_{I}\right)\right)$ is $\alpha$-efficient if the functional $V_{\alpha}$ : $\left(\mathbb{R}_{+} \times L^{1}(\mathcal{P})_{+}\right)^{I} \rightarrow \mathbb{R}$ achieves the maximum over the set of allocations $\Lambda(e, E)=$ $\left\{(c, C) \in\left(\mathbb{R}_{+} \times L^{1}(\mathcal{P})_{+}\right)^{I}: \sum\left(c_{i}, C_{i}\right) \leq(e, E) \mathcal{P}\right.$-q.s. $\}$.

Under concavity of the utility functionals, $\alpha$-efficiency for some $\alpha \in \Delta_{I}$ is equivalent to Pareto optimality, while this is related to an equilibrium with transfer payment. As a first step we establish the existence of $\alpha$-efficient allocations.

Theorem 1 Suppose $V_{i}: \mathbb{R}_{+} \times L^{1}(\mathcal{P})_{+} \rightarrow \mathbb{R}, i \in \mathbb{I}$, are utility functionals given by (2) with a concave utility index, then there exists an $\alpha$-efficient allocation. If each $\mathfrak{c}_{i}$ is linear, the solution correspondence

$$
\mathbb{C}(\alpha, e, E)=\underset{(x, X) \in \Lambda(e, E)}{\arg \max } \sum_{i \in \mathbb{I}} \alpha_{i} V_{i}\left(x_{i}, X_{i}\right)
$$

is nonempty, convex and weakly compact valued. Moreover, if for each $(t, i) \in$ $\{0, T\} \times \mathbb{I}, u_{i}^{t}$ is twice continuously differentiable, i.e. $u_{i}^{t} \in C^{2,1}\left(\mathbb{R}_{+} ; \mathbb{R}\right)$, there is a continuous selection $(c, C) \in \mathbb{C}$, such that $\alpha \mapsto C_{i}(\alpha, E)$ is continuously differentiable on $\AA_{I}$. In particular, we have a

$$
\mu \in \bigcap_{i \in \mathbb{I}} \alpha_{i} \partial U_{i}\left(C_{i}(\alpha, E)\right) \neq \emptyset, \quad \text { where } \mathrm{d} \mu=\alpha_{i} u_{i}^{T^{\prime}}\left(C_{i}(\alpha, E)\right) \mathrm{d} P
$$

The result is interesting in its own right, but will play as well a central role in the approach to the existence of an (analytic) equilibrium. From the theorem we immediately infer that there is a fully insured efficient allocation, when the aggregate endowment is certain, i.e $E(\omega)$ is constant $\mathcal{P}$-quasi surely.

If the aggregate endowment is uncertain but unambiguous, i.e. $E \in \mathrm{M}[\mathcal{P}]$, structural properties of optimal allocations depend additionally on preferences. The following example illustrates how Pareto sharing rules determine the insurance properties and the resulting net trades.

\footnotetext{
${ }^{39}$ We assume that the agents in the economy share the same set of priors, but they do not agree via their ambiguity index. A simple generalization could be a heterogeneity in the ambiguity via a modification of equivalent priors with a bounded density.
} 
Example 4 Let the uncertainty model be that of Example 1 and the aggregate endowment of the economy be unambiguous, i.e. $E \in \mathrm{M}[\mathcal{P}]$ and by Corollary 2, we have $E=E_{T}=\mathbb{E}^{\mathcal{P}}[E]+\int_{0}^{T} \theta_{t}^{E} \mathrm{~d} B_{t}^{G}$, for some adapted and integrable process $\theta^{E}$. Note, that the individual endowment is still allowed to be ambiguous. Now suppose for each $i \in \mathbb{I}$ that the functional form of optimal consumption $C_{i}(\alpha, \cdot) \in C^{2,1}\left(\mathbb{R}_{+}\right)$ is twice continuously differentiable and not linear, which holds if each $u_{i} \in C^{3,1}\left(\mathbb{R}_{+}\right)$ has a nonlinear risk tolerance, for details see Hara, Huang, and Kuzmics (2007). This implies $C_{i}^{\prime \prime}(\alpha, \cdot) \neq 0$ and we derive for each $i \in I$ by the G-Itô formuld

$$
C_{i}\left(\alpha, E_{T}\right)=C_{i}\left(\alpha, \mathbb{E}^{\mathcal{P}}[E]\right)+\int_{0}^{T} C_{i}^{\prime}\left(\alpha, E_{t}\right) \theta_{t}^{E} \mathrm{~d} B_{t}^{G}+\frac{1}{2} \int_{0}^{T} C_{i}^{\prime \prime}\left(\alpha, E_{t}\right)\left(\theta_{t}^{E}\right)^{2} \mathrm{~d}\left\langle B^{G}\right\rangle_{t} .
$$

Due to the nonzero $\mathrm{d}\left\langle B^{G}\right\rangle$-part, we have $C_{i}(\alpha, E) \notin \mathrm{M}[\mathcal{P}]$ by Corollary 2. This means that the Pareto optimal allocation is ambiguous. In case of linear risk tolerance, i.e. $C_{i}^{\prime \prime}(\alpha, \cdot)=0$, the same computation imply an unambiguous Pareto optimal allocation. From this we infer that the absence of idiosyncratic ambiguity does not always leads to unambiguous efficient allocations.

Concerning the net trades $\xi_{i}=C_{i}(\alpha, E)-E_{i}$, we have, unless the "pathological" case that the $\mathrm{d}\left\langle B^{G}\right\rangle$-part of $E_{i}$ eliminates the $\mathrm{d}\left\langle B^{G}\right\rangle$-part of $C_{i}(\alpha, E)$, ambiguous net trades, meaning that $\xi_{i} \notin \mathrm{M}[\mathcal{P}]$.

In the case of linear risk tolerance a sufficient condition for unambiguous net trades is $E_{i} \in \mathrm{M}[\mathcal{P}]$, for each agent $i \in \mathbb{I}$.

Comparing this example with De Castro and Chateauneuf (2011), we see that an unambiguous aggregate endowment is not sufficient to observe an unambiguous Pareto optimal allocation. The missing gap relies on the structure of the sharing rule. Note that the arguments in the present setting are based on results from stochastic analysis under $G$-expectation.

\subsubsection{The General Equilibrium}

Now we introduce the classical notion of an Arrow-Debreu equilibrium. Note that, the feasibility holds $\mathcal{P}$-quasi surely and for the price functional we require $c_{1, \mathcal{P}^{-}}$ continuity as discussed in Section 2.1. By Proposition $1, L^{1}(\mathcal{P})$ is a Banach lattice, hence positive and linear functionals on $L^{1}(\mathcal{P})$ are automatically $c_{1, \mathcal{P}}$-continuous. The $I+1$-tuple $\left(\left(\bar{c}_{1}, \bar{C}_{1}\right), \ldots,\left(\bar{c}_{I}, \bar{C}_{I}\right) ;(\pi, \Pi)\right) \in\left(\mathbb{R}_{+} \times L^{1}(\mathcal{P})_{+}\right)^{I} \times\left(\mathbb{R} \times L^{1}(\mathcal{P})^{*}\right)$ consisting of a feasible allocation and a continuous linear price functional, is called a contingent Arrow-Debreu equilibrium, if

1. For all $i,\left(\bar{c}_{i}, \bar{C}_{i}\right)$ maximizes $V_{i}$ on $\mathbb{R}_{+} \times L^{1}(\mathcal{P})_{+}$under $\Psi\left(c-e_{i}, C-E_{i}\right) \leq 0$.

2. The allocation $(\bar{c}, \bar{C})$ is feasible: $\sum_{i \in \mathbb{I}}\left(\bar{c}_{i}, \bar{C}_{i}\right)=(e, E), \quad \mathcal{P}$-q.s.

Next, we reconsider the utility gradient of the agent when she faces a maximization problem in terms of a first order condition. As in the single prior setting, the excess utility map encodes the "universal system of equations" of the defined equilibrium. In matters of the utility maximization, the particular form of the gradient causes

${ }^{40}$ The result can be found in Peng $(2010)$. 
a modification in the definition of the excess utility map, see Appendix A.2 for the details of the construction method. In general, the gradient is an element of the topological dual. The representation of the dual space, see Proposition 2 and Lemma 1, implies $D U_{i}(C) \in \partial U_{i}(C) \subset L^{1}(\mathcal{P})^{*}$, where a supergradient can be represented by $D U_{i}(C)(h)=\mathrm{E}^{P}\left[u_{i}^{\prime}(C) h\right]$, for some $P \in M_{i}(C)$ and direction $h \in L^{1}(\mathcal{P})$.

Remark 4 In infinite dimensional commodity spaces, the positive cone may have an empty interior. In this situation, a properness condition is needed to establish the existence of an equilibrium. Note that by Proposition $1, L^{1}(\mathcal{P})$ is an order continuous Banach lattice. As we aim to establish an equilibrium allocation with an explicit dependency of the effective priors, we only mention this whole branch of abstract existence result. We refer to Martins-da Rocha and Riedel (2010) and the references therein.

In order to connect the gradient with the price system, in terms of Theorem 1 and the second fundamental theorem of welfare economics, we have to make an assumption on the integrability of $u^{\prime}(E)$.

Assumption 3 Let the aggregate endowment $E \in L^{1}(\mathcal{P})_{+}$be strictly positive $\mathcal{P}$-q.s. and let $e=\sum e_{i}>0$. We assum $£^{41}$

$$
\max _{\alpha \in \Delta_{I}}\left(u_{\alpha}^{0^{\prime}}(e)+u_{\alpha}^{T^{\prime}}(E)\right) \in L^{\infty}(\mathcal{P}) \quad \text { and } \quad \bigcap_{i \in \mathbb{I}} \operatorname{dom}\left(\mathfrak{c}_{i}\right) \neq \emptyset
$$

This assumption is closely related to a cone condition, which is important for the existence of an equilibrium in infinite dimensional commodity spaces, see also Remark 2.2 in Dana (1993). Moreover it guarantees that the price system is an element of the semi-strict order dual $L^{1}(\mathcal{P})_{\oplus}^{*}$, see Subsection 3.1 for details. The proof of the following theorem is based on the gross substitute property of the modified excess utility map $\Phi: \Delta_{I} \times \mathcal{P} \rightarrow \mathbb{R}^{I}$, see Definition 5 in Appendix A.2. In order to guarantee this property we have to make the following well-known assumption.

Assumption 4 For each $(i, t) \in \mathbb{I} \times\{0, T\}, x \mapsto x \cdot u_{i}^{t^{\prime}}(x)$ is non-decreasing.

The assumption is equivalent to the Arrow-Pratt measure of relative risk-aversion being less or equal than one, when $u_{i}^{t^{\prime}}$ is twice differentiable. We are ready to state the first main result of the paper.

Theorem 2 Suppose each agent satisfies the conditions of Lemma 1, with strictly concave and strictly monotone utility index and a linear penalty term $\mathfrak{c}_{i}$. Under Assumption 1-4 there is a Pareto optimal Arrow-Debreu equilibrium $\left(c_{1}^{*}, C_{1}^{*}, \ldots, c_{I}^{*}, C_{I}^{*} ;(\pi, \Pi)\right)$, with $\Pi \in L^{1}(\mathcal{P})_{\oplus}^{*}$.

The Pareto optimal equilibrium allocation is based on an $\alpha^{*}$-efficient weighting $\alpha^{*} \in \Delta_{I}$, so that we denote the set of equilibrium priors by

$$
\mathcal{P}_{E} \subset \mathbb{P}\left(\alpha^{*}\right) \subset \mathcal{P}
$$

\footnotetext{
${ }^{41}$ Fix $t \in\{0, T\}$ and $\alpha \in \Delta_{I}, u_{\alpha}^{t}: \mathbb{R}_{++} \rightarrow \mathbb{R}$ is given by $u_{\alpha}^{t}(e)=\max _{x \in \Lambda(e, 0)} \sum \alpha_{i} u_{i}^{t}\left(x_{i}\right)$. Here $L^{\infty}(\mathcal{P})$ is the closure of $\mathcal{C}_{b}$ under the norm $c_{\infty, \mathcal{P}}(X)=\inf \{M \geq 0:|X| \leq M, \mathcal{P}-$ q.s. $\}$. See again Denis, Hu, and Peng (2011) for more details.
} 
This set of common unadjusted priors $\mathbb{P}\left(\alpha^{*}\right)$ is constructed in Appendix A.2, see also Subsection 2.1 for an illustration of the construction idea. One important property is that the representative agent behaves as an agent with variational utility. In the following, we illustrate in the sense in which $\alpha$-efficient allocations are uniquely specified. Namely, under every equilibrium prior $P \in \mathcal{P}_{E}$ an equilibrium allocation is determined $P$-a.s. To illustrate this point in more detail, we define a different allocation resulting in the same utility. As the following example points out, the reasoning is consistent with the finite-dimensional example in Section 2.1, where the Leontief-type utility of the agents created a similar degree of freedom. This is illustrated in Figure 2 (b).

Example 5 Consider an economy with two agents $i=1,2$ under the uncertainty model of Example 1 and Remark 3.2. Utilities are given by

$$
U_{1}(C)=\min _{P \in \mathcal{P}} \mathrm{E}^{P}[\ln (C)]=-E_{G}[\ln (C)] \quad \text { and } U_{2}(C)=\min _{P \in \mathcal{P}} \mathrm{E}^{P}\left[C^{1 / 2}\right] .
$$

The endowment of each agent is a function of the $G$-Brownian motion at time $T$, i.e. $E_{i}=\varphi_{i}\left(B_{T}^{G}\right) \in L^{1}(\mathcal{P})_{+}$, where $\varphi_{i}: \mathbb{R} \rightarrow \mathbb{R}_{+}$is assumed to be convex, so that $\varphi_{i}=\exp$ is in principle a possible choice. Moreover, let $\varphi=\varphi_{1}+\varphi_{2}$ so that the aggregate endowment can be written as a function of the $G$-Brownian motion $B^{G}$, i.e $E=\varphi\left(B_{T}^{G}\right)$. After some computation an equilibrium consumption allocation $C_{i}(\alpha, E)=\Upsilon_{i}^{\alpha}\left(B_{T}^{G}\right)$ is given by

$$
\Upsilon_{1}^{\alpha}\left(B_{T}^{G}\right)=\frac{2 \cdot \varphi\left(B_{T}^{G}\right)}{1+\sqrt{1+\varphi\left(B_{T}^{G}\right) \bar{\alpha}^{2}}}, \quad \Upsilon_{2}^{\alpha}\left(B_{T}^{G}\right)=\left(\frac{\bar{\alpha} \cdot \varphi\left(B_{T}^{G}\right)}{1+\sqrt{1+\varphi\left(B_{T}^{G}\right) \bar{\alpha}^{2}}}\right)^{2},
$$

where $\alpha=\alpha_{1}, 1-\alpha=\alpha_{2}$ and $\bar{\alpha}=\frac{\alpha}{1-\alpha}$. Since $\Upsilon_{1}^{\alpha}\left(B_{T}^{G}\right)+\Upsilon_{2}^{\alpha}\left(B_{T}^{G}\right)=\varphi\left(B_{T}^{G}\right)$ holds $\mathcal{P}$-q.s., this results into a feasible allocation. Since $\Upsilon_{i}^{\alpha}=C_{i}(\alpha, \cdot) \circ \varphi$ and $C_{2}(\alpha, \cdot)$ is convex and increasing, we have that $\Upsilon_{\alpha}^{2}$ is convex as well. In order to observe the effective prior, note that $u_{2}\left(C_{2}(\alpha, E)\right)=u_{2}\left(\Upsilon_{2}^{\alpha}\left(B_{T}^{G}\right)\right)$ is concave, which implies $M_{2}\left(C_{2}(\alpha, E)\right)=\left\{P^{\bar{\sigma}}\right\}=\mathcal{P}_{E}$ by the following computation:

We discuss the optimal allocation via tools from stochastic analysis under the $G$ expectation. Suppose that each optimal consumption has the complete representation property of Remark 3.2. 2 we can write

$$
\Upsilon_{2}^{\alpha}\left(B_{T}^{G}\right)=E_{G}\left[\Upsilon_{\alpha}^{2}\left(B_{T}^{G}\right)\right]+\int_{0}^{T} \theta_{t}^{2} \mathrm{~d} B_{t}^{G}-\int_{0}^{T} G\left(\eta_{t}^{2}\right) \mathrm{d} t+\frac{1}{2} \int_{0}^{T} \eta_{t}^{2} \mathrm{~d}\left\langle B^{G}\right\rangle_{t}
$$

where $\theta_{t}^{2}=f_{x}^{2}\left(t, B_{t}^{G}\right), \eta_{t}^{2}=f_{x x}^{2}\left(t, B_{t}^{G}\right)$ and $f^{2}\left(T, B_{T}^{G}\right)=\Upsilon_{\alpha}^{2}\left(B_{T}^{G}\right)=C_{2}(\alpha, E)$. As illustrated in the first part of the example $\Upsilon_{\alpha}^{2}$ is convex and by Section 1 in Chapter II of Peng (2010) it follows that $f^{2}(t, \cdot): \mathbb{R} \rightarrow \mathbb{R}_{+}$is convex for each $t \in[0, T]$.

\footnotetext{
${ }^{42} \mathrm{~A}$ sufficient condition is the boundedness of $\partial_{x} \Upsilon_{i}^{\alpha}(x)$ on $\mathbb{R}_{+}$.
} 
Hence $f_{x x}^{2} \geq 0$ and we deduce that the last two terms of (4) can be written as

$$
\begin{aligned}
-K_{T}^{2} & =-\int_{0}^{T} G\left(f_{x x}^{2}\left(t, B_{t}^{G}\right)\right) \mathrm{d} t+\frac{1}{2} \int_{0}^{T} f_{x x}^{2}\left(t, B_{t}^{G}\right) \mathrm{d}\left\langle B^{G}\right\rangle_{t} \\
& =-\frac{1}{2} \int_{0}^{T} \sup _{\sigma \in[\underline{\sigma}, \bar{\sigma}]} \sigma f_{x x}^{2}\left(t, B_{t}^{G}\right)+\hat{a}_{t} f_{x x}^{2}\left(t, B_{t}^{G}\right) \mathrm{d} t \\
& =\frac{1}{2} \int_{0}^{T}\left(\hat{a}_{t}-\bar{\sigma}\right) f_{x x}^{2}\left(t, B_{t}^{G}\right) \mathrm{d} t,
\end{aligned}
$$

The martingale representation theorem also tells us, that the process $\left(-K_{t}^{2}\right)$ is a $G$-martingale. Moreover, we have $-K_{t}^{2} \equiv 0 P^{\bar{\sigma}}$-a.s. and $-K_{t}^{2} \neq 0$ under every other prior in $\mathcal{P} \backslash\left\{P^{\bar{\sigma}}\right\}$.

With this observation, we construct a different allocation having the same utility. Consider $\bar{\eta}=\varepsilon \cdot \eta^{2}, \varepsilon \in(0,1)$. We show that the allocation $\left(C_{1}(\alpha, E)+\right.$ $\left.\varepsilon K_{T}^{2}, C_{2}(\alpha, E)-\varepsilon K_{T}^{2}\right)$ is also $\alpha$-efficient and satisfies $C_{1}(\alpha, E) \neq \bar{C}_{1}:=C_{1}(\alpha, E)+$ $\varepsilon K_{T}^{2} P$-a.s. for every $P \in \mathcal{P} \backslash\left\{P^{\bar{\sigma}}\right\}$.

Since, $\hat{a}_{t} \in[\underline{\sigma}, \bar{\sigma}]$, it follows that $K_{t}^{2} \geq 0 \mathcal{P}$-q.s. Hence, by the monotonicity of the utility functional, this reallocation does not worsen the utility of agent 1, i.e. $U_{1}\left(C_{1}(\alpha, E)+\varepsilon K_{T}^{2}\right) \geq U_{1}\left(C_{1}(\alpha, E)\right.$.

For agent 2, the positive homogeneity of $G$ implies $G(\bar{\eta})=\varepsilon G(\eta)$. From this we see that $P^{\bar{\sigma}}$ is still the only effective prior with respect to $\bar{C}_{1}$, since $\frac{1+\varepsilon}{2} \int_{0}^{T}\left(\bar{\sigma}-\hat{a}_{t}\right) \eta_{t}^{2} d t=$ $-(1+\varepsilon) K_{T}^{2}=-\bar{K}_{T}^{2}$ yields

$$
\bar{C}_{2}=E_{G}\left[\Upsilon_{\alpha}^{2}\left(B_{T}^{G}\right)\right]+\int_{0}^{T} Z_{t}^{2} d B_{t}^{G}-(1+\varepsilon) K_{T}^{2}
$$

Specifically, under $P^{\bar{\sigma}}$ the compensation term satisfies $\bar{K}^{2} \equiv 0$ and hence the utility of agent 2 remains unaffected, i.e. $U_{2}\left(C_{2}(\alpha, E)\right)=U_{2}\left(\bar{C}^{2}\right)$. Note that, for $\varepsilon$ sufficiently small, we have $P^{\bar{\sigma}} \in M_{2}\left(\bar{C}_{2}\right)$, since $M_{2}\left(C_{2}(\alpha, E)\right)=\left\{P^{\bar{\sigma}}\right\}$.

Finally, we state the semi-strictly positive equilibrium price system given by $X \mapsto$ $\Pi(X)=\mathrm{E}^{P^{\bar{\sigma}}}\left[u_{\alpha^{*}}^{\prime}(E) \cdot X\right]$, where the effective prior is induced by

$$
\arg \min _{P \in \mathcal{P}} \mathrm{E}^{P}\left[u_{\alpha^{*}}(E)\right]=\left\{P^{\bar{\sigma}}\right\}=\mathbb{P}\left(\alpha^{*}\right)=\mathcal{P}_{E} .
$$

Ambiguity aversion creates the worst case prior $P^{\bar{\sigma}}$ and the density part in terms of risk attitudes is given by

$$
u_{\alpha}^{\prime}(E)=\frac{\alpha}{\varphi\left(B_{T}^{G}\right)}\left(1+\sqrt{1+\varphi\left(B_{T}^{G}\right) \bar{\alpha}^{2}}\right) .
$$

Summing up, we have illustrated how the new martingale representation theorem can be applied to construct many different efficient allocations and analyze their structural properties.

Note that the convexity of $\Upsilon_{\alpha}^{2}(\cdot)$ induces the unique effective prior $P^{\bar{\sigma}}$, which can be seen as an extreme case. Different effective priors corresponding to more complex volatility specifications depend in general on the structure of the efficient sharing rules, see again Example 4 for the most simplest case. 


\subsection{The Existence of Incomplete Security Markets}

With the dynamics of the uncertainty model of Section 4.1, we are now in the position to formulate trading processes and the Radner equilibrium. Before, we introduce an assumption for the space of consumption profiles at time $T$. This gives us a certain invariance on the space of net trades.

Assumption 5 The density part of the equilibrium utility gradients at time $T$, $u_{\alpha}^{T^{\prime}}(E)$ is bounded away from zero, i.e.

$$
\varepsilon<\max _{\alpha \in \Delta_{I}} u_{\alpha}^{T^{\prime}}(E) \quad \mathcal{P} \text {-q.s., for some } \varepsilon>0 .
$$

This assumption is satisfied when the aggregate endowment is bounded away from zero and the utility functions $u_{i}$ satisfy the Inada condition at zero. It guarantees the boundedness above and below away from zero of our state price density $\psi=u_{\alpha^{*}}^{T}(E)$, where $\alpha^{*}$ is the equilibrium weight of Theorem 2 in Subsection 4.2. It follows that $L^{1}(\mathcal{Q})=L^{1}(\mathcal{P})$, where

$$
\mathcal{Q}=\left\{Q: \mathrm{d} Q=\frac{\psi}{\mathrm{E}^{P}[\psi]} \mathrm{d} P, \text { for some } P \in \mathcal{P}\right\} .
$$

This invariance is of importance, since the density $\psi$ is derived from the equilibrium and is not a primitive. ${ }^{43}$ Based on the set of unadjusted equilibrium priors $\mathcal{P}_{E}$, we denote by $\mathcal{Q}_{E}=\left\{Q: \mathrm{d} Q=\frac{\psi}{\mathrm{E}^{P}[\psi]} \mathrm{d} P, P \in \mathcal{P}_{E}\right\}$ the set of equilibrium pricing measures.

Now, we introduce a Radner equilibrium of prices, plans and price expectation related to the present mutually singular prior model. The price process $S=\left(S_{t}\right)_{t \in[0, T]}$ for our long lived security is a $\mathcal{P}$-semimartingale ${ }^{44}$ on the filtered sublinear expectation space $\left(\Omega, L^{1}(\mathcal{P}), \mathbb{E}^{\mathcal{P}}, \mathbb{F}\right)$.

As we have seen in Example 4, we observe unambiguous net trades when strong assumptions on endowments and utilities are imposed. By the martingale representation theorem under $\mathcal{Q}$ applied with respect to $\mathcal{Q}$-ambiguous net trades, i.e. $C_{i}-E_{i} \notin \mathrm{M}[\mathcal{Q}]$, a disposal term $K_{T}^{i}$ appears. To account for this useless consumption, where the equilibrium price system is zero, we allow for a gain process with a feasible possibility of free disposal of wealth under non equilibrium priors. This is achieved in terms of a security with possibly negative dividend under some $Q \in \mathcal{Q}$. We consider the set of admissible trading processes already mentioned in Corollary 2, with certain regularity conditions:

1. Well defined: $\theta$ is $\mathbb{F}$-predictable and $\mathbb{E}^{\mathcal{P}}\left[\int_{0}^{T} \theta_{t}^{2} \mathrm{~d}\langle S\rangle_{t}\right]<\infty .45$

2. Gain process: $\int \theta \mathrm{d} S$ is a $\mathcal{P}$-q.s. well defined stochastic integral.

\footnotetext{
${ }^{43}$ In Section 3 of Duffie and Huang (1985) a similar assumption can be found.

${ }^{44}$ As defined in Pham and Zhang (2012), the uncertain process $S$ is a $\mathcal{P}$-semimartingale if it is a $P$-semimartingale for every $P \in \mathcal{P}$. Note that their Assumption 4.1 is in the present setting fulfilled, since we augment the filtration with the $\mathcal{P}$-polar sets.

${ }^{45}$ The bracket process is given by $\langle B\rangle=B^{2}-\int B \mathrm{~d} B$, where the stochastic integral is defined pathwise, see Soner, Touzi, and Zhang (2012b) and the references therein.
} 
3. Self-financing: The trading process satisfies the accounting identity

$$
X_{t}^{\theta}=\theta_{t} S_{t}=\theta_{0} S_{0}+\int_{0}^{t} \theta_{r} \mathrm{~d} S_{r}, \quad \mathcal{P} \text {-q.s. for every } t \in[0, T] .
$$

The space of processes $\theta$ satisfying conditions 1.-3. is denoted by $\Theta(S)$. From Corollary 2 it follows directly that the gain process must be a symmetric martingale in order to establish a perfect hedge. If we consider the volatility uncertainty as a robustness constraint, the corollary just characterizes a perfect hedging portfolio with some initial value if and only if the terminal payoff is unambiguous. We come now to the formal definition of a Radner equilibrium under volatility uncertainty.

Definition $4 A$ Radner equilibrium for $\mathcal{E}(e, E)$ is comprised of $N+1$ long lived security claiming $D=\left(D^{0}, \ldots, D^{N}\right) \in L^{1}(\mathcal{P})^{N+1}$, with price process $S=$ $\left(S^{0}, \ldots, S^{N}\right)$, a set of trading strategies $\theta^{i} \in \Theta(S), i \in \mathbb{I}$ and a price $\pi>0$ for consumption at time zero, which satisfies:

For each agent $i \in \mathbb{I}$, the consumption $\left(e_{i}-X_{0}^{\theta^{i}} \pi^{-1}, E_{i}+X_{T}^{\theta^{i}}\right)$ maximizes $V_{i}: \mathbb{R}_{+} \times L^{1}(\mathcal{P})_{+} \rightarrow \mathbb{R}$ on the budget set

$$
B\left(e_{i}, E_{i}, \pi, D, S\right)=\left\{\left(e_{i}-X_{0}^{\theta} \pi^{-1}, E_{i}+X_{T}^{\theta}\right) \in \mathbb{R}_{+} \times L^{1}(\mathcal{P})_{+}: \theta \in \Theta(S)\right\},
$$

so that the market clears, $\sum_{i \in \mathbb{I}} \theta_{t}^{i}=0$ P-q.s., for every $t \in[0, T]$.

A priori, the functional capability of the financial market as a mechanism is reflected by the marketed space $\mathrm{M}[\mathcal{Q}]$. In comparison to the single prior case, market completeness is not an intrinsic property in terms of the simpler all-encompassing martingale representation.

Theorem 3 Suppose the security-spot economy

$$
\mathcal{E}(e, E)=\left\{(\Omega, \mathcal{F}, \mathbb{F}, \mathcal{P}), D,\left\{V_{i}, \mathbb{R}_{+} \times L^{1}(\mathcal{P}),\left(e_{i}, E_{i}\right)\right\}_{i \in \mathbb{I}}\right\}
$$

satisfies Assumptions 1 to 5, then there is a Radner equilibrium for $\mathcal{E}(e, E),\left(\left\{\left(\theta^{i}\right)\right\}_{i \in \mathbb{I}}, \pi, D, S\right)$, which implements the given Arrow-Debreu Equilibrium $\left(\left\{c_{i}, C_{i}\right\}_{i \in \mathbb{I}} ; \mathrm{E}^{P}[\psi \cdot]\right)$ if and only if

$$
\mathbb{E}^{\mathcal{P}}\left[\psi\left(C_{i}-E_{i}\right)\right]=\mathrm{E}^{P}\left[\psi\left(C_{i}-E_{i}\right)\right], \quad \text { for every } i \in \mathbb{I} .
$$

In this case, we have:

1. The financial market is effectively dynamically complete.

2. Trade may be ambiguous, i.e. $\left(C_{i}-E_{i}\right) \notin \mathrm{M}[\mathcal{Q}]$.

3. Under the equilibrium prior, each $\left(c_{i}, C_{i}\right)$ is perfectly hedged.

The condition in (5) states a relation about endogenous objects. More precisely, (5) can be understood as the existence of a worst case prior $Q$ as an element of $\mathcal{Q}_{E}$. 
At the same time, $Q$ must be a maximizing prior with respect to the uncertaintyadjusted sublinear expectation $\mathbb{E}^{\mathcal{Q}}$ evaluated at each net trade $\xi_{i}=C_{i}-E_{i}$, i.e.

$$
Q \in \mathcal{Q}\left(\xi_{i}\right)=\arg \max _{Q \in \mathcal{Q}} \mathrm{E}^{Q}\left[\xi_{i}\right]
$$

Note that if the net trades are unambiguous then this condition is automatically satisfied, see Example 4 and 5.

In the presence of volatility uncertainty, the proof of Theorem 3 can be regarded as a canonical extension of Duffie and Huang (1985). In their example with Brownian Noise only two long lived security price processes are required to admit a complete Radner equilibrium. This follows from the two summands in the (Brownian) martingale representation. The present volatility uncertainty setup requires a third component in the martingale representation. This is a compensation part of disposal under non maximizing priors $\mathcal{Q}\left(\xi_{i}\right){ }^{46}$ For this reason, we observe a martingale multiplicity of three. But Theorem 3 also tells us, that in contrast to the single prior case, the implementation of efficient Arrow-Debreu equilbria into a Radner equilibrium is not always possible. The Pareto efficiency of the Radner equilibrium is quite surprising, since multiple period incomplete markets are typically only constrained efficient. Nevertheless, the efficiency still depends on which equilibrium allocation is considered.

Under a non-equilibrium prior $P \in \mathcal{P} \backslash \mathcal{P}_{E}$ the consumption profiles are for some agents superhedged. However, under the priors

$$
P^{\prime} \in M_{i}\left(C_{i}\right) \cap\left\{P \in \mathcal{P}: \mathrm{d} P=\psi^{-1} \mathrm{~d} Q, Q \in \mathcal{Q}\left(\xi_{i}\right)\right\},
$$

the hedge is still perfect, i.e. $K^{P^{\prime}, i}=0 P^{\prime} \otimes \mathrm{d} t$-a.e. Under such priors, the deflated gain process becomes a martingale and under every other effective prior only a supermartingale. This is still consistent with the "no expected gain from trade" hypothesis of Duffie (1986). The following example illustrates under the $G$-framework, how the Radner equilibrium incorporates with the new component in the martingale representation theorem. The dynamics of the price process of the new security, obtained from Lemma 3 in Appendix A.2, get a more explicit specification. Again, the price process depends heavily on the net trades of the Arrow-Debreu equilibrium.

Example 6 Apart from the condition in Theorem 3, suppose we are in the Gframework and every net trade $\xi_{i}=C_{i}-E_{i}, i \in \mathbb{I}$, has the complete martingale representation property, see Remark 3.2. Denoting by $\hat{a}_{t}=\frac{\mathrm{d}}{\mathrm{d} t}\left\langle B^{G}\right\rangle_{t}$ the time derivative of the quadratic variation of the $G$-Brownian motion, we have

$$
\begin{aligned}
K_{t}^{i} & =\int_{0}^{t} G\left(\eta_{t}^{i}\right) \mathrm{d} t-\frac{1}{2} \int_{0}^{t} \eta_{t}^{i} \mathrm{~d}\left\langle B^{G}\right\rangle_{t} \\
& =\int_{0}^{t} \sup _{\sigma \in[\underline{\sigma}, \bar{\sigma}]} \sigma \eta_{t}^{i} \mathrm{~d} t-\int_{0}^{t} \eta_{t}^{i} \hat{a}_{t} \mathrm{~d} t=\frac{1}{2} \int_{0}^{t} \sigma_{t}^{i} \eta_{t}^{i} \mathrm{~d} t-\frac{1}{2} \int_{0}^{t} \eta_{t}^{i} \hat{a}_{t} \mathrm{~d} t \\
& =\frac{1}{2} \int_{0}^{t}\left(\sigma_{t}^{i}-\hat{a}_{t}\right) \cdot \eta_{t}^{i} \mathrm{~d} t
\end{aligned}
$$

\footnotetext{
${ }^{46}$ Alternatively to this particular and novel security, we could introduce a family of securities being contingent on the prior. However, such a prior-dependent contingency would stand in opposition to the present quasi-sure analysis in aggregated terms.
} 
for some $\eta^{i}$, as an output of the complete martingale representation. In the derivation, each process $\sigma^{i}$ corresponds to a $P^{\sigma^{i}} \in \mathcal{P}$ with $P^{\sigma^{i}} \sim Q_{i} \in \mathcal{Q}$. Similarly to the proof of Theorem 3, let us consider the following dividend structure

$$
D^{0} \equiv 1, \quad D^{1}=B_{T}^{G}, \quad D^{2}=\int_{0}^{T}-\frac{1}{2} \sum_{i \in \mathbb{I}}\left(\sigma_{t}^{i}-\hat{a}_{t}\right) \mathrm{d} t .
$$

The price process $S_{t}^{2}=\mathbb{E}_{t}^{\mathcal{Q}}\left[D^{2}\right]$ for the asset with dividend $D^{2}$ and the portfolio processes of each agent are $\mathbb{E}^{\mathcal{Q}}$-martingales, i.e. a $Q$-supermatingale under every prior $Q \in \mathcal{Q}$ and a $Q$-martingale under some $Q \in \mathcal{Q}$ corresponding to the no gain from trade hypothesis. Moreover, the price process is absolutely continuous with respect to $\mathrm{d} t$ and given by

$$
\mathrm{d} S_{t}^{2}=-\frac{1}{2} \sum_{i \in \mathbb{I}}\left(\sigma_{t}^{i}-\hat{a}_{t}\right) \mathrm{d} t, \quad S_{0}^{2}=0 .
$$

For the strategy of this bounded variation security $S^{2}$, consider the following partition of unity $\sum_{i \in \mathbb{I}} \kappa_{t}^{i}=1 \mathcal{Q}$-q.e. so that

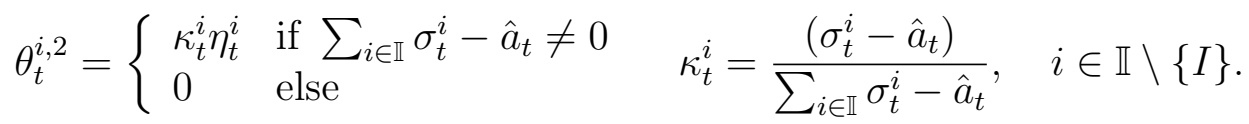

Note that in the uncertainty neutral world $\mathcal{Q}$ the asset price $S^{2}$, so that $K^{i}=$ $\int \theta^{i, 2} \mathrm{~d} S^{2}$, may become negative under some non equilibrium measures $\mathcal{Q} \backslash\{Q\}$, where $\mathrm{d} Q=\psi \mathrm{d} P$ and $P \in \mathcal{P}_{E}$ satisfies (5). ${ }^{47}$ In essence this depends on the net trade and the equilibrium expectation. Remember, the compensation (or disposal) part in the martingale representation prefigures this possibility. This can be understood as the interplay between the $\mathcal{P}$-q.s. clearing condition and the disposal parts of the net trades. For instance, this phenomenon is not present, when net trades are unambiguous and induces portfolio processes being symmetric $\mathcal{Q}$-martingales without a disposal part.

\section{A Appendix}

The first part of the appendix collects the proofs of Section 2. First we review some convergence results used, and which are relevant especially to the proof for Lemma 1.

Convergence properties of sublinear expectations, Denis, Hu, and Peng (2011):

1. Let $\left\{P_{n}\right\}_{n \in \mathbb{N}} \subset \mathcal{P}$ converges weakly to $P \in \mathcal{P}$. Then, for each $X \in L^{1}(\mathcal{P})$, we have $\mathrm{E}^{P_{n}}[X] \rightarrow \mathrm{E}^{P}[X]$.

2. Let $\mathcal{P}$ be weakly compact and let $\left\{X_{n}\right\}_{n \in \mathbb{N}} \subset L^{1}(\mathcal{P})$ be such that $X_{n} \searrow X$, then $\mathbb{E}^{\mathcal{P}}\left[X_{n}\right] \searrow \mathbb{E}^{\mathcal{P}}[X]$.

\footnotetext{
${ }^{47}$ In the situation of Example 5 this is possible, since $\sigma^{i} \equiv \bar{\sigma}>\hat{a}$. However, the same is true for $S_{1}$. In each case, a splitting of the positive and negative parts would guarantee positive price processes.
} 
3. Let $\left(X_{n}\right)_{n \in \mathbb{N}}$ be a sequence in $L^{1}(\mathcal{P})$ which converges to $X$ in $L^{1}(\mathcal{P})$. Then there exists a subsequence $\left(X_{n_{k}}\right)_{k \in \mathbb{N}}$ which converges to $X$ quasi-surely in the sense that it converges to $X$ outside a $\mathcal{P}$-polar set.

We say a sequence $\left(X_{n}\right)_{n \in \mathbb{N}}$ converges in capacity to $X$ if for each $\varepsilon>0$ we have $\sup _{P \in \mathcal{P}} P\left(\left|X_{n}-X\right|>\varepsilon\right)$ convergeing to zero.

Hierarchy of convergence, Cohen, Ji, and Peng (2011): Quasi sure convergence implies convergence in capacity.

Dominated convergence for sublinear expectation, $\mathrm{Xu}(2010)$ : Let $\left(X_{n}\right)_{n \in \mathbb{N}}$ be a sequence in $L^{1}(\mathcal{P})$ such that $\left|X_{n}\right| \leq Y \in L^{1}(\mathcal{P})$, for each $n \in \mathbb{N}$. If $X_{n} \rightarrow X$ in capacity, then $\lim _{n \rightarrow \infty} \mathbb{E}^{\mathcal{P}}\left[X_{n}\right]=\mathbb{E}^{\mathcal{P}}[X]$.

In our multiple prior setting quasi sure convergence does not imply convergence in capacity, see the Appendix of $\mathrm{Xu}(2010)$ for an example. In this case, the limit $X$ is not necessarily an element of $L^{1}(\mathcal{P})$.

\section{A.1 A 1: Details and Proofs of Section 3}

As mentioned in Subsection 2.1, two random variables $X, Y \in L^{1}(\mathcal{P})$ can be distinguished if there is a prior $P \in \mathcal{P}$ such that $P(X \neq Y)>0$. Such null elements are characterized by random variables which are $\mathcal{P}$-polar. $\mathcal{P}$-polar sets which are evaluated under every prior are zero or one, although, the value may differ between different priors. A property holds quasi-surely (q.s.) if it holds outside a polar set. Furthermore, the space $L^{1}(\mathcal{P})$ is characterized in Denis, Hu, and Peng (2011) via

$$
L^{1}(\mathcal{P})=\left\{X \in L(\Omega): X \text { has a q.c. version, } \lim _{n \rightarrow \infty} \mathbb{E}^{\mathcal{P}}\left[|X| 1_{\{|X|>n\}}\right]=0\right\} .
$$

A mapping $X: \Omega \rightarrow \mathbb{R}$ is said to be quasi-continuous (q.c.) if for all $\varepsilon>0$ there exists an open set $O$ with $c(O)=\sup _{P \in \mathcal{P}} P(O)<\varepsilon$ such that $\left.X\right|_{O^{c}}$ is continuous. We say that $X: \Omega \rightarrow \mathbb{R}$ has a q.c. version if there exists a quasi-continuous function $Y: \Omega \rightarrow \mathbb{R}$ with $X=Y$ q.s.

Proof of Proposition 1 We show $\inf (X, Y)=X \wedge Y \in L^{1}(\mathcal{P})$ for every $X, Y \in$ $L^{1}(\mathcal{P})$ via the representation in (6). Since $\{|X|>n\} \supset\{|X \wedge Y|>n\}$, we have by the sublinearity of $\mathbb{E}^{\mathcal{P}}$

$$
\mathbb{E}^{\mathcal{P}}\left[|X \wedge Y| 1_{\{|X \wedge Y|>n\}}\right] \leq \mathbb{E}^{\mathcal{P}}\left[|X| 1_{\{|X|>n\}}\right]+\mathbb{E}^{\mathcal{P}}\left[|Y| 1_{\{|Y|>n\}}\right] \underset{n \rightarrow \infty}{\longrightarrow} 0 .
$$

Since $X$ and $Y$ have a q.c. version, there are $\bar{\varepsilon}, \varepsilon_{X}, \varepsilon_{Y}>0$ such that $\varepsilon_{X}+\varepsilon_{Y}<\bar{\varepsilon}$ with $c\left(O_{X}\right)<\varepsilon_{X}, c\left(O_{Y}\right)<\varepsilon_{Y}$ and hence $c\left(O_{X} \cup O_{Y}\right) \leq c\left(O_{X}\right)+c\left(O_{Y}\right)<\bar{\varepsilon}$. Because $\left.X\right|_{\left(O_{X} \cup O_{Y}\right)^{c}}$ and $\left.Y\right|_{\left(O_{X} \cup O_{Y}\right)^{c}}$ are both continuous, the quasi-continuity of $X \wedge Y$ follows. The order relation is indeed a lattice operation.

That $L^{1}(\mathcal{P})$ is a Banach space is shown in Denis, Hu, and Peng (2011). $L^{1}(\mathcal{P})$ is a Banach lattice, since for all $X, Y \in L^{1}(\mathcal{P})$ with $|X| \leq|Y|$, i.e. $|X| \leq|Y| P$-a.s. for all $P \in \mathcal{P}$ imply

$$
c_{1, \mathcal{P}}(X)=\max _{P \in \mathcal{P}} \mathrm{E}^{P}[|X|]=\mathrm{E}^{P^{\prime}}[|X|] \leq \mathrm{E}^{P^{\prime}}[|Y|] \leq c_{1, \mathcal{P}}(Y)
$$


for some maximizing prior $P^{\prime}$ for $c_{1, \mathcal{P}}(X)$. Fix a sequence of positive random variables $\left(X_{n}\right)$ in $L^{1}(\mathcal{P})$ such that $X_{n} \searrow 0$ in $L^{1}(\mathcal{P})$. Hence $X_{1}$ dominates the sequence and an application of the dominated convergence under sublinear expectation gives us

$$
\lim _{n \rightarrow \infty} c_{1, \mathcal{P}}\left(X_{n}\right)=\lim _{n \rightarrow \infty} \mathbb{E}^{\mathcal{P}}\left[\left|X_{n}\right|\right]=\mathbb{E}^{\mathcal{P}}\left[\left|\lim _{n \rightarrow \infty} X_{n}\right|\right]=0
$$

Hence, $L^{1}(\mathcal{P})$ is an order continuous Banach lattice.

Proof of Proposition 2 In our construction, the underlying sublinear expectation space is given by $\left(\Omega, \mathcal{C}_{b}(\Omega), \mathbb{E}^{\mathcal{P}}\right)$, as given by Theorems 25 and 52 in Denis, Hu, and Peng (2011) $L^{1}(\mathcal{P})=L_{G}^{1}(\Omega)$. Since $\Omega$ is a polish space and $\mathcal{P}$ is a weakly compact by Assumption $1, c_{1, \mathcal{P}}$ is a Prokhorov capacity.

If $l: \tilde{L}^{1}(\mathcal{P}) \rightarrow \mathbb{R}$ is a non-negative linear functional, then there is a non-negative measure $\mu$ with support $\Omega$ such that

$$
l(X)=\int X \mathrm{~d} \mu, \quad \text { for every } X \in L^{1}(\mathcal{P}),
$$

This is shown in Proposition 11 of Feyel and de La Pradelle (1989). In Theorem 6 by Feyel and De La Pradelle (1977), it is shown that every continuous linear functional is the difference of two non-negative linear functionals.

$\tilde{L}^{1}(\mathcal{P})$ is given by the space of $c_{1, \mathcal{P}}$-equivalence classes of $\overline{\mathcal{C}}_{b}(\Omega){ }^{c_{1, \mathcal{P}}}$, so that the domain is modified via the so called Lebesgue prolongation. The explicit representation of the $c_{1, \mathcal{P}}$-topological dual of $\tilde{L}^{1}(\mathcal{P})$, can be found in the first chapter of Kervarec (2008), Theorem I.30.

Proof of Corollary 1 " $\Rightarrow$ ": By Proposition 2, we have $l(X)=\mathrm{E}^{P}[\psi X]$, since $\psi>0$ P-a.s and $P(X>0)>0$, therefore $l(X)>0$ follows.

$" \Leftarrow ": l \in L^{1}(\mathcal{P})^{*}$ implies again by Proposition 2 that we can write $l(X)=\mathrm{E}^{P}[\psi X]$. Suppose $\psi \notin L^{\infty}(P)_{++}$then $P(\psi>0, X>0)=0$ for some $P \in \mathcal{P}$ is possible. We have a contradiction.

Proof of Lemma 1 1. Monotonicity follows directly from the monotonicity of the utility index $u$. Let $P_{X} \in M(X) \subset \mathcal{P}$ be a minimizing prior of $U(X)$. Semi-strict monotonicity follows from $u^{\prime}(X)>0$ on a set with a positive measure with respect to $P_{X}$ and

$$
U(X+Z)-U(X) \geq \mathrm{E}^{P_{X}}[u(X+Z)-u(X)]>\mathrm{E}^{P_{X}}\left[u^{\prime}(X+Z) \cdot Z\right] \geq 0,
$$

where the strict inequality follows from strict concavity of $u$ and $P(Z>0)>0$ for every $P \in \mathcal{P}$.

2. The mapping $C \mapsto \mathrm{E}^{P}[u(C)]+\mathfrak{c}(P)$ is concave for each $P \in \mathcal{P}$ and the inf operation preserves concavity. We prove the strict concavity on $\mathrm{M}[\mathcal{P}]$. Let $\alpha \in(0,1)$ and $C, X \in L^{1}(\mathcal{P})_{+} \cap \mathrm{M}[\mathcal{P}]$, with $C \neq X$. We compute

$$
\begin{aligned}
\alpha U(C)+(1-\alpha) U(X) & \leq \min _{P \in \mathcal{P}} \mathrm{E}^{P}[\alpha u(C)+(1-\alpha) u(X)]+\mathfrak{c}(P) \\
& <\min _{P \in \mathcal{P}} \mathrm{E}^{P}[u(\alpha C+(1-\alpha) X)]+\mathfrak{c}(P) \\
& =U(\alpha C+(1-\alpha) X),
\end{aligned}
$$


where the first inequality follows from the concavity of $C \mapsto U(C)$.

The functional $U$ is not strictly concave on its whole domain, since $C \neq X$ in $L^{1}(\mathcal{P})$ does not imply $C \neq X$ under every $P \in \mathcal{P}$, hence one can easily pick two elements $C$ and $X$ which are $P$-a.s. equal, where $P \in M(\lambda C+(1-\lambda) X)$ and deduce $a$ contradiction, when following the proof of concavity.

3. Let the sequence $\left(X_{n}\right)_{n \in \mathbb{N}} \subset L^{1}(\mathcal{P})_{+}$converges to $X$ in $L^{1}(\mathcal{P})$. In order to prove the assertion, we show that every subsequence $\left(Y_{n_{k}}\right)_{k \in \mathbb{N}}$ of $\left(X_{n}\right)$ has in turn a subsequence $\left(Z_{n}\right)_{n \in \mathbb{N}}$ such that

$$
\limsup _{n \rightarrow \infty} U\left(Z_{n}\right) \leq U(X)
$$

Let $P^{X} \in M(X)$ be a minimizing prior and $\left(Y_{n_{k}}\right)_{k \in \mathbb{N}}$ be a subsequence of $\left(X_{n}\right)_{n \in \mathbb{N}}$. There is a subsequence $\left(Z_{n}\right)_{n \in \mathbb{N}}$ in $\left(Y_{n_{k}}\right)_{k \in \mathbb{N}}$ and some $Z \in L_{+}^{1}(\mathcal{P})$ satisfying

$$
Z_{n}(\omega) \rightarrow X(\omega) \text { and } 0 \leq Z_{n}(\omega) \leq Z(\omega) \text { for } P^{X} \text {-a.s. }
$$

We may take $Z=X+\sum_{n \in \mathbb{N}}\left|Z_{n+1}-Z_{n}\right|$, with $c_{1, \mathcal{P}}\left(Z_{n+1}-Z_{n}\right) \leq 2^{-n}$. Monotonicity of $u$ implies $0 \leq u\left(Z_{n}(\omega)\right) \leq u(Z(\omega))$ and $u\left(Z_{n}(\omega)\right) \rightarrow u(X(\omega))$ for $P^{X}$ almost every $\omega \in \Omega$.

So, by the limsup-version of Fatou's lemma under $P^{X}$ we deduce

$$
\begin{aligned}
\limsup _{n \rightarrow \infty} U\left(Z_{n}\right) & \leq \limsup _{n \rightarrow \infty} \mathrm{E}^{P^{X}}\left[u\left(Z_{n}\right)\right]+\mathfrak{c}\left(P^{X}\right) \\
& \leq \mathrm{E}^{P^{X}}[u(X)]+\mathfrak{c}\left(P^{X}\right)=U(X) .
\end{aligned}
$$

We prove the norm continuity of $U$, when $\mathfrak{c}$ is linear. To show $U\left(X_{n}\right) \rightarrow U(X)$ for some norm convergent sequence $\left(X_{n}\right)_{n \in \mathbb{N}}$, it suffices again to show that every subsequence of $\left(X_{n}\right)_{n \in \mathbb{N}}$ has a subsequence $\left(Z_{n}\right)_{n \in \mathbb{N}}$ with $U\left(Z_{n}\right) \rightarrow U(X)$.

Let $\left(X_{n_{k}}\right)_{k \in \mathbb{N}}$ be a subsequence of $\left(X_{n}\right)_{n \in \mathbb{N}}$. We have $X_{n_{k}} \rightarrow X$ in $c_{1, \mathcal{P}}$. There is a subsequence $\left(Z_{n}\right)_{n \in \mathbb{N}}$ of $\left(X_{n_{k}}\right)_{k \in \mathbb{N}}$ and a $Z \in L^{1}(\mathcal{P})$ with $0 \leq Z_{n} \leq Z \mathcal{P}$-q.s. and $Z_{n} \rightarrow X \mathcal{P}$-q.s., which implies convergence in capacity, (see the beginning of Appendix A). We may take $Z$ as before. By the monotonicity and continuity of $u$, we have $0 \leq u\left(Z_{n}\right) \leq u(Z) \mathcal{P}$-q.s. and $u\left(Z_{n}\right) \rightarrow u(X) \mathcal{P}$-q.s. An application of dominated convergence under sublinear expectation, as stated in the beginning of Appendix A, gives us

$$
\begin{aligned}
\lim _{n \rightarrow \infty} U\left(Z_{n}\right) & =-\mathbb{E}^{\mathcal{P}}\left[\phi-u\left(\lim _{n \rightarrow \infty} Z_{n}\right)\right] \\
& =-\mathbb{E}^{\mathcal{P}}[\phi-u(X)]=\min _{P \in \mathcal{P}} \mathrm{E}^{P}[u(X)]-\langle P, \phi\rangle=U(X) .
\end{aligned}
$$

This implies the $c_{1, \mathcal{P}}$-continuity of the utility functional. Note that the lower semicontinuity and linearity of the penalty term implies continuity, hence we can find a $\phi \in L^{1}(\mathcal{P})$, to give a representation in terms of a bilinear form.

4. That $\mathcal{P}$ is also $\sigma\left(L^{1}(\mathcal{P}), L^{1}(\mathcal{P})^{*}\right)$-weakly compact follows by the same arguments as in the proof of Proposition 2.4 in Bion-Nadal and Kervarec (2012), since it is a closed subset of the nonnegative part of the unit ball of $L^{1}(\mathcal{P})^{*}$. Effective priors exist, since $P \mapsto \mathrm{E}^{P}[X]$ is weakly continuous for every $X \in L^{1}(\mathcal{P})$, and build a 
convex weakly compact subset of $\mathcal{P}$.

Let $P^{*} \in M(C)$ be an effective prior for $C$ and let $X \in L^{1}(\mathcal{P})_{+}$be arbitrary. By the concavity and differentiability of the utiltiy index $u$, this implies

$$
\begin{aligned}
U(X)-U(C) & =\min _{P \in \mathcal{P}} \mathrm{E}^{P}[u(X)]+\mathfrak{c}(P)-\min _{P \in \mathcal{P}} \mathrm{E}^{P}[u(C)]+\mathfrak{c}(P) \\
& \leq \mathrm{E}^{P^{*}}[u(X)]+\mathfrak{c}\left(P^{*}\right)-\mathrm{E}^{P^{*}}[u(C)]+\mathfrak{c}\left(P^{*}\right) \\
& \leq \mathrm{E}^{P^{*}}\left[u^{\prime}(C)(X-C)\right] .
\end{aligned}
$$

The characterization of the superdifferential follows from the fact that $\partial U(X) \subset$ $L^{1}(\mathcal{P})^{*}$, Proposition 2 and Corollary 2 of Theorem 2.8.2 in Clarke (1990).

\section{A.2 A 2: Details and Proofs of Section 4}

Proof of Lemma 2 By Assumption 1 the set of priors is convex, weakly compact and stable under pasting. The semigroup property of the conditional expectations can be found in Proposition 3.6 (i) of Nutz and Soner (2012).

Alternatively, when the set $\mathcal{D}$ is given by an explicit correspondence process, one can also apply Theorem 2.6 form Epstein and Ji (2013b).

Proof of Corollary 2 The alternative representation is an application of the martingale representation theorem in Section 4.2. It can be easily verified that $\mathrm{M}[\mathcal{P}]$ is a closed subspace of $L^{1}(\mathcal{P})$. Unambiguous random variables can be identified as the terminal value of the stochastic integral, which is the image of a linear operator, with preimage $\Theta(B)$.

In order to concentrate on the essential difficulties of the proofs in Subsection 4.2, we do not consider consumption and endowments at time 0 , until the proof of Theorem 3. The product structure of the consumption profiles and the additive utility imply that proofs within the two period economy are slight generalizations. To do so we identify $\Lambda(0, E)$ with $\Lambda(E)=\left\{C \in L^{1}(\mathcal{P})_{+}^{I}: \sum C_{i} \leq E\right\}$.

Proof of Theorem 1 The functional $U_{\alpha}: \Lambda(E) \rightarrow \mathbb{R}$ is weakly upper semicontinuous, by Lemma 1. By Proposition $1, L^{1}(\mathcal{P})$ is a Banach lattice with order continuous norm. This implies that the order interval $[[0, E]]=\left\{x \in L^{1}(\mathcal{P}): 0 \leq x \leq E\right\}$ is $\sigma\left(L^{1}(\mathcal{P}), L^{1}(\mathcal{P})^{*}\right)$-compact, this result can be found in Theorem 2.3 .8 of Aliprantis, Brown, and Burkinshaw (1990) and Section 2 in Yannelis (1991). Hence, $\Lambda(E)$ is $\sigma\left(L^{1}(\mathcal{P})^{I},\left(L^{1}(\mathcal{P})^{I}\right)^{*}\right)$-compact, as a closed subset of $[[0, E]]^{I}$ under the same topology. The Weierstrass Theorem (Theorem 2.43 in Aliprantis and Border (2006)) implies the existence of a maximizer.

The upper hemicontinuity of $\mathbb{C}(\alpha, \cdot): \Delta_{I} \times L^{1}(\mathcal{P})_{+} \Rightarrow L^{1}(\mathcal{P})_{+}^{I}$ follows from Berge Maximum theorem, where each $U^{i}$ is continuous. We prove the existence of a continuously differentiable selection. The well defined mapping $C(\alpha, e): \Delta_{I} \times \mathbb{R}_{+} \rightarrow \mathbb{R}_{+}^{I}$ is the unique solution of the pointwise problem

$$
C(\alpha, e)=\underset{x_{i} \geq 0, \sum x_{i} \leq e}{\operatorname{argmax}} \sum \alpha_{i} u_{i}\left(x_{i}\right), \quad(\alpha, e) \in \Delta_{I} \times \mathbb{R}_{+},
$$


which is continuously differentiable on $\AA_{I} \times \mathbb{R}_{++}$, the interior of $\operatorname{dom}(C)$. For every $\alpha \in \Delta_{I}$ there is a $P \in \mathcal{P}$ such that the modified economy with $\operatorname{dom}\left(\tilde{\mathfrak{c}}_{i}\right)=\{P\}, i \in \mathbb{I}$, satisfies the same first order condition as in the original economy

$$
\mu \in L^{1}(\mathcal{P})^{*}, \quad \mathrm{~d} \mu=u_{\alpha}^{\prime}(E) \mathrm{d} P=\alpha_{i} u_{i}^{\prime}\left(C_{i}(\alpha, E)\right) \mathrm{d} P
$$

for every $i \in \mathbb{I}$ such that $\alpha_{i} \neq 0$. This implies the $\alpha$-efficiency of $\left\{C_{i}(\alpha, E)\right\}_{i \in \mathbb{I}}$ in the original and $\tilde{\mathfrak{c}}_{i}$-modified economy. Feasibility holds by construction. Hence, $C \in \mathbb{C}$ is a continuously differentiable selection in $\alpha$.

The proof of Theorem 2 needs some preparation and is divided into the following four propositions, which make use of the conditions for Theorem 2. The proof strategy of Theorem 2 adapts the ideas of Section 3 in Dana (2004). With the existence of an $\alpha$-efficient allocation from Theorem 1, we can consider the single-valued solution selection $C: \Delta_{I} \times L^{1}(\mathcal{P})_{+} \rightarrow L^{1}(\mathcal{P})_{+}^{I}$ of the concave program $\left(U_{\alpha}, \Lambda(E)\right)$, given by

$$
\left\{C_{i}(\alpha, E)\right\}_{i \in \mathbb{I}} \in \underset{\left(C_{i}\right) \in \Lambda(E)}{\arg \max } \sum_{i \in \mathbb{I}} \alpha_{i} U_{i}\left(C_{i}\right)
$$

in the next steps. Now, we introduce our excess utility map. In comparison to the classical case, the mapping has to be modified, since the utility gradient cannot be solely represented by a random variable with a conjugate integrability order. In general for some $X \in L^{1}(\mathcal{P})_{+}$, the set of effective priors $M(X)$ is not unique, since it is the minimizer of a convex (and not strictly convex) program, i.e. Gateaux differentiability is in general not true. Hence, we propose a prior dependency in the excess utility to account for this change in the universal system of equations.

Proposition 3 Under Assumptions 1-4 with $\operatorname{dom}\left(\mathfrak{c}_{i}\right)=\{P\}$, for all $i \in \mathbb{I}$, there is a P-a.s. unique equilibrium.

As illustrated in Example 5, in general there is no hope for a $\mathcal{P}$-q.s. unique equilibrium.

Proof of Proposition 3 By Theorem 1, for each $\alpha \in \Delta_{I}$, a unique $\alpha$-efficient allocation exists. The proof now follows the lines of Dana (1993), where the present commodity price duality is given by $\left\langle L^{1}(\mathcal{P}), L^{\infty}(P)\right\rangle$. Here, the continuity of the excess utility map follows by the dominated convergence result at the beginning of Appendix A.

Let us denote by $\mathbb{G} \mathbb{E}: \mathcal{P} \rightarrow \Delta_{I}$, the single-valued correspondence which asserts to every prior the unique equilibrium weight $\alpha^{P}$ of the relevant vNM-economy $(P)$ in Proposition 3. This motivates the following definition.

Definition 5 Let $C_{i}(\alpha, E)$ be the argmax of an $\alpha$-efficient allocation with von Neumann Morgenstern utility under $P \in \mathcal{P}$. The excess utility map $\Phi: \Delta_{I} \times \mathcal{P} \rightarrow \mathbb{R}^{I}$ is given by

$$
\Phi_{i}(\alpha, P)=\alpha_{i}^{-1} \mathrm{E}^{P}\left[u_{\alpha}^{\prime}(E) \cdot\left(C_{i}(\alpha, E)-E_{i}\right)\right], \quad i \in \mathbb{I} .
$$


The primitives of the economy specify this modified excess utility map. A zero for the standard excess utility map, when only the utility weight $\alpha$ is the variable, guarantees an equilibrium. The modification in the definition is caused by the equilibrium prior, a new component in the universal system of equations. Due to the first order conditions of individual maximization, this object appears because of the given structure of the topological dual space.

A zero $(\alpha, P) \in \Delta_{I} \times \mathcal{P}$ of $\Phi$ is not sufficient to guarantee an equilibrium, since an arbitrary $P \in \mathcal{P}$ may not lie in the set of common effective priors $\bigcap_{i \in \mathbb{I}} M_{i}\left(C_{i}(\alpha, E)\right)$, where the consumption $C_{i}(\alpha, E)$ is taken from the $\alpha$-efficient allocation. To account for this situation, we need the following two Propositions.

First, we prove that at every Pareto optimal allocation, the intersection of the risk adjusted effective prior is not empty. As we will see below, this ensures that the excess utility map can attain a zero in $\mathbb{R}^{I}$ on the appropriate set of priors. To do so, we reformulate $\alpha$-efficiency in terms of a supremal convolution from convex analysis. For $E \in L^{1}(\mathcal{P})$, let

$$
\square_{i \in \mathbb{I}} \alpha_{i} U_{i}(E)=\max _{\sum C_{i}=E} \sum \alpha_{i} U_{i}\left(C_{i}\right),
$$

and denote the superdifferential of $\square_{i=1}^{I} \alpha_{i} U_{i}$ by $\partial \square U_{\alpha}$, see Laurent (1972) for details. Note that the domain of each $U_{i}$ equals $L^{1}(\mathcal{P})_{+}$.

The following proposition states that for $\alpha$-efficient allocations the utility supergradients of the agents agree. We also discuss the $\alpha$-dependency of common effective priors.

Proposition 4 1. Let $\left(C_{1}(\alpha, E), \ldots, C_{I}(\alpha, E)\right) \in \Lambda(E)$ be the $\alpha$-efficient allocation of Theorem 1. We get

$$
\bigcap_{i \in \mathbb{I}} \partial \alpha_{i} U_{i}\left(C_{i}(\alpha, E)\right)=\partial \square U_{\alpha}(E) \neq \emptyset
$$

for some $\alpha \in \Delta_{I}$. Moreover, the set $\partial \square U_{\alpha}(E)$ is weakly compact and convex.

2. The set of common risk unadjusted priors $\mathbb{P}(\alpha)=\cap_{i \in \mathbb{I}} M_{i}\left(C_{i}(\alpha, E)\right)$ satisfies,

$$
\begin{aligned}
\mathbb{P}(\alpha) & =\left\{P \in \mathcal{P}: \exists \mu \in \partial \square U_{\alpha}(E) \text { with } \mathrm{d} \mu=u_{\alpha}^{\prime}(E) \mathrm{d} P\right\} \\
& =\arg \min _{P \in \mathcal{P}} \mathrm{E}^{P}\left[u_{\alpha}(E)-\sum_{i \in \mathbb{I}} \alpha_{i} \phi_{i}\right]
\end{aligned}
$$

where $\phi_{i}$ is the representation of the linear penalty term $\mathfrak{c}_{i}$.

3. The correspondence $\mathbb{P}: \Delta_{I} \rightarrow \mathcal{P}$ is upper hemicontinuous on $\Delta_{I}$. Moreover, $\mathbb{P}$ is weakly compact and convex valued.

Proof of Proposition 41 . The allocation $\left(C_{1}(\alpha, E), \ldots, C_{I}(\alpha, E)\right) \in \Lambda(E)$ can be related to an $\alpha$-weighted program $\left(U^{\alpha}, \Lambda(E)\right)$. We formulate this in terms of supremal convolution. By construction we have $\sum_{i} C_{I}(\alpha, E)=E \mathcal{P}$-q.s. and

$$
\square_{i \in \mathbb{I}} \alpha_{i} U_{i}(E)=\sum \alpha_{i} U_{i}\left(C_{i}(\alpha, E)\right)
$$


By Lemma 1, we have $\partial U_{i}\left(C_{I}(\alpha, E)\right) \neq \emptyset$, for each $i \in \mathbb{I}$. The first part of the proposition follows from Proposition 6.6.4 in Laurent (1972). The convexity of $\partial \square U^{\alpha}(E)$ can be found in Theorem $47 A$ in Zeidler (1985). The intersection of compact sets is again compact.

2. Let $\bar{P} \in \mathbb{P}(\alpha)$, we derive

$$
\begin{aligned}
& \max _{(X) \in \Lambda(E)} \sum_{i \in \mathbb{I}} \alpha_{i} U_{i}\left(X_{i}\right)=\sum_{i \in \mathbb{I}} \alpha_{i} \min _{P \in \mathcal{P}} \mathrm{E}^{P}\left[u_{i}\left(C_{i}(\alpha, E)-\phi_{i}\right]\right. \\
= & \mathrm{E}^{\bar{P}}\left[\sum_{i \in \mathbb{I}} \alpha_{i}\left(u_{i}\left(C_{i}(\alpha, E)\right)-\phi_{i}\right)\right]=\min _{P \in \mathcal{P}} \mathrm{E}^{P}\left[u_{\alpha}(E)-\sum_{i \in \mathbb{I}} \alpha_{i} \phi_{i}\right],
\end{aligned}
$$

where the pointwise definition of $u_{\alpha}$ can be found in the footnote in Assumption 3. The result follows from Lemma 1.4 .

3. The upper hemicontinuity of the correspondence $\mathbb{P}$ follows from Berge's maximum theorem with respect to the $\alpha$-parametrized and linear problem $\min _{P \in \mathcal{P}} \mathrm{E}^{P}\left[u_{\alpha}(E)-\right.$ $\left.\sum \alpha_{i} \phi_{i}\right]$. The values are weakly compact and convex, due to the first part of the proposition.

In the next step, we relate Proposition 3 with our notion of excess utility.

Proposition 5 The tuple $\left(\left\{C_{i}\left(\alpha^{*}, E\right)\right\}_{i \in \mathbb{I}}, \mathrm{E}^{P^{*}}\left[u_{\alpha^{*}}^{\prime}(E) \cdot\right]\right)$ is an Arrow-Debreu equilibrium if and only if

$$
\Phi\left(\alpha^{*}, P^{*}\right)=0 \text { and } P^{*} \in \mathbb{P}\left(\alpha^{*}\right),
$$

which is equivalent to $(\alpha, P) \in \operatorname{gr}\left(\mathbb{G} \mathbb{E}^{-1}\right) \cap \operatorname{gr}(\mathbb{P})$ or $P \in \mathbb{P} \circ \mathbb{G} \mathbb{E}(P)$.

Proof of Proposition $5 " \Leftarrow "$ : Each prior $P \in \mathbb{P}(\alpha)$ is associated to a supergradient $D U_{i}\left(C_{i}\right)(X)=\mathrm{E}^{P}\left[u_{i}^{\prime}\left(C_{i}\right) X\right]$ for each agent $i \in \mathbb{I}$ simultaneously. A possible prior $P \notin \mathbb{P}(\alpha)$ with a zero in the excess demand is not related to at least one agent $k$ 's first order condition with a positive weight $\alpha_{k}$. Whereas, if $\Phi$ is not zero, we have only an equilibrium with transfer payment.

$" \Rightarrow ":$ By Proposition 4, $\Phi\left(\alpha^{*}, P^{*}\right)=0$ and $P^{*} \in \mathbb{P}\left(\alpha^{*}\right)$ implies the existence of an equilibrium $\left(\left\{C_{i}^{P^{*}}\left(\alpha^{*}, E\right)\right\}_{i \in \mathbb{I}}, u_{\alpha^{*}}^{\prime}(E)\right)$ under $v N M\left(P^{*}\right)$ utility, i.e. $X \mapsto$ $\mathrm{E}^{P^{*}}\left[u_{i}(X)\right]-c_{i}$ under $P^{*} \in \mathbb{P}\left(\alpha^{*}\right)$, where $c_{i}=E^{P^{*}}\left[\phi_{i}\right]$ and $C_{i}^{P}(\alpha, \cdot)$ corresponds to the $\alpha$-efficient consumption of agent $i$ under $v N M(P)$ utility. We get

$$
\begin{array}{ll} 
& \mathrm{E}^{P^{*}}\left[u_{\alpha^{*}}^{\prime}(E)\left(C-E_{i}\right)\right] \leq 0 \\
\text { implies } & \mathrm{E}^{P^{*}}\left[u_{i}(C)\right]-c_{i} \leq \mathrm{E}^{P^{*}}\left[u_{i}\left(C_{i}^{P^{*}}\left(\alpha^{*}, E\right)\right)\right]-c_{i} .
\end{array}
$$

This implies $U_{i}(C) \leq U_{i}\left(C_{i}^{P^{*}}\left(\alpha^{*}, E\right)\right)$, due to $U_{i}(C) \leq \mathrm{E}^{P^{*}}\left[u_{i}(C)-\phi_{i}\right]$. Hence, $\left(\left\{C_{i}^{P^{*}}\left(\alpha^{*}, E\right)\right\}_{i \in \mathbb{I}}, \mathrm{E}^{P^{*}}\left[u_{\alpha^{*}}^{\prime}(E) \cdot\right]\right.$ is an equilibrium of the original economy.

Agent $i$ 's set of effective priors $M_{i}\left(C_{i}(\alpha, E)\right) \supset \mathbb{P}(\alpha)$ at an optimal consumption forms the basis for the set of equilibrium priors. The first order condition of $\alpha$ efficient allocations relies on the set of common supergradient $\partial \square U_{\alpha}$. The risk adjustment via the normalized marginal utility $u_{\alpha}^{\prime}(E)$ of the representative agent delivers the correct set of equilibrium priors, see Proposition 4.2. This is consistent with the decomposition of the linear price systems and the modified excess utility map. 
Proof of Theorem 2 Define the functional $\rho: \Delta_{I} \times \mathcal{P} \rightarrow \mathbb{R}$ by

$$
\rho(\alpha, P)=\min _{i \in \mathbb{I}} \Phi_{i}(\alpha, P)
$$

By Proposition 6.2 $\Phi_{i}(\alpha, \cdot)$ is linear and weakly continuous, hence $\rho(\alpha, \cdot)$ is weakly continuous. From an application of Proposition 6.1 we follow for each $P \in \mathcal{P}$ the continuity of $\rho(\cdot, P)$, since the pointwise infimum of continuous functions is again continuous. Since the maximum of $\rho(\cdot, P)$ over $\Delta_{I}$ is by construction a zero, the solution mapping $\mathbb{G} \mathbb{E}$ is also given by

$$
\mathbb{G} \mathbb{E}(P)=\arg \max _{\alpha \in \Delta_{I}} \rho(\alpha, P) .
$$

Therefore by Berge's maximum theorem, $\mathbb{G E}$ is a single-valued and upperhemicontinuous correspondence and hence continuous when viewed as a function. Now, $\mathbb{P} \circ \mathbb{G E}: \mathcal{P} \rightarrow \mathcal{P}$ is a composition of upper hemicontinuous correspondences and hence again upper hemicontinuous. By Proposition 4.1., $\mathbb{P}$ is convex and weakly compact valued and hence so is $\mathbb{P} \circ \mathbb{G} \mathbb{E}$. Since the vector space of signed measure on $(\Omega, \mathcal{F})$ equipped with the topology of weak convergence is a locally convex topological vector space, we apply the Kakutani-Glicksberg-Fan fixed point theorem (Theorem 17.55 in Aliprantis and Border (2006)) with respect to $\mathbb{P} \circ \mathbb{G} \mathbb{E}$, and the result follows by Proposition 5.

Proposition 6 1. For each $P \in \mathcal{P}$, the function $\Phi(\cdot, P)$ is continuous in the interior of $\Delta_{I}$ and $\|\Phi(\alpha, P)\|_{\mathbb{R}^{I}} \rightarrow+\infty$ whenever $\alpha_{i} \rightarrow 0$ for some $i \in \mathbb{I}$.

2. For each $\alpha \in \Delta_{I}$, the function $\Phi(\alpha, \cdot)$ is weakly continuous.

The following result is used in the proof of Theorem 2.

Proof of Proposition 6 1. This follows from Proposition 3 and the continuous differentiability of each $u_{i}$. Since $P \in \mathcal{P}$ is fixed, the limit behavior follows by same argument as in the the standard single prior case.

2. Let $\left\{P_{n}\right\}_{n \in \mathbb{N}}$ be a sequence in $\mathcal{P}$ which converges weakly to some prior $P$. According to the first result at the beginning of Appendix A,

$$
\lim _{n \rightarrow \infty} \mathrm{E}^{P_{n}}\left[u_{\alpha}^{\prime}(E) \cdot\left(C_{i}(\alpha, E)-E_{i}\right)\right]=\mathrm{E}^{P}\left[u_{\alpha}^{\prime}(E) \cdot\left(C_{i}(\alpha, E)-E_{i}\right)\right]
$$

and which proves continuity in the weak topology.

The equilibrium weight $\alpha^{*}$ relates the residual set of priors by $\mathcal{P}_{E}=\mathbb{P}\left(\alpha^{*}\right)$.

\section{A.2.1 Proofs of Subsection 4.3}

By $\mathcal{Q}$-q.e., we denote $\mathcal{Q} \otimes \mathrm{d} t=\{\mathbb{Q} \otimes \mathrm{d} t, \mathbb{Q} \in \mathcal{Q}\}$-quasi everywhere.

Proof of Theorem 3 We begin with the only if part of the theorem and denote $\xi_{i}=C_{i}-E_{i}$. Suppose there is an agent $i \in \mathbb{I}$, such that $\mathcal{Q}_{E} \cap \mathcal{Q}\left(\xi_{i}\right)=\emptyset 4$ This

\footnotetext{
${ }^{48}$ Here, $\mathcal{Q}(X)=\arg \max _{Q \in \mathcal{Q}} \mathrm{E}^{Q}[X]$ denotes the effective (uncertainty adjusted) priors under the sublinear equilibrium expectation.
} 
implies $\mathrm{E}^{Q}\left[\xi_{i}\right]<\mathbb{E}^{\mathcal{Q}}\left[\xi_{i}\right]$, where $Q \in \mathcal{Q}_{E}$. In order to guarantee the implementation of the Arrow-Debreu equilibrium, the portfolio process $X^{\theta^{i}}$ with $\theta^{i} \in \Theta(S)$ requires $X_{T}^{\theta^{i}}=X_{0}^{\theta^{i}}+\int_{0}^{T} \theta_{t}^{i} \mathrm{~d} S_{t}$ and must satisfy

$$
X_{T}^{\theta^{i}}=\xi \quad \text { and } \quad X_{0}^{\theta^{i}}=\pi\left(e_{i}-c_{i}\right) .
$$

An application of the martingale representation theorem to $\xi_{i}$ implies $X_{0}^{\theta^{i}}=\mathbb{E}^{\mathcal{Q}}\left[\xi_{i}\right]$, so that the only constants in the martingale representation and the self-financing condition must be equal. On the other side, we have by the Arrow-Debreu budget set $X_{0}^{\theta^{i}}=\pi\left(e_{i}-c_{i}\right)=\mathrm{E}^{Q}\left[\xi_{i}\right]$, which is a contradiction to $\mathcal{Q}_{E} \cap \mathcal{Q}\left(\xi_{i}\right) \neq \emptyset$ or equivalently to (5) in the formulation Theorem 3.

To proof the other direction, fix the following elements in $L^{1}(\mathcal{P})$ as the dividend of the first two securities:

$$
D^{0} \equiv 1, \quad D^{1}=B_{T}, \quad D^{2}=K_{T},
$$

where $K_{T}$ is specified in step two below by virtue of Lemma 3. We introduce the candidates for the price of consumption at time zero and the price process of the security. Let the price of $D$ at time $t$ be $S_{t}^{1}=\mathbb{E}_{t}^{\mathcal{Q}}\left[D^{1}\right]$ and $S_{t}^{0}=\mathbb{E}_{t}^{\mathcal{Q}}\left[D^{0}\right]=1$. The positive scalar $\pi$ is the price of time zero consumption. We divide the proof into four steps. In the first step and second, we introduce the candidate trading strategies for agent $i \in \mathbb{I} \backslash\{I\}$ and show market clearing in the third step. The last step shows that the trading strategies are maximal elements in the budget sets.

1. Let $\xi_{i} \in L^{1}(\mathcal{P}), i \in \mathbb{I} \backslash\{I\}$, be some feasible net trades. The process

$$
X_{t}^{i}=\mathbb{E}_{t}^{\mathcal{Q}}\left[\xi_{i}\right]-\mathbb{E}^{\mathcal{Q}}\left[\xi_{i}\right], \quad t \in[0, T]
$$

is an integrable $\mathcal{Q}$-martingale and we have by the martingale representation

$$
X_{t}^{i}=\int_{0}^{t} \theta_{r}^{i, 1} \mathrm{~d} S_{r}^{1}-K_{t}^{i},
$$

Q-q.e. Fix some strategy $\theta^{i}:=\left(\theta^{i, 0}, \theta^{i, 1}, \theta^{i, 2}\right) \in \Theta\left(S^{0}, S^{1}, S^{2}\right)=: \Theta(S)$, where $\theta^{i, 0}$ and $\theta^{i, 2}$ are specified in step two.

As a candidate Radner equilibrium allocation at time $T$, we consider the allocation generated by the Arrow-Debreu equilibrium allocation, i.e. $\xi_{i}=\left(\bar{C}_{i}-E_{i}\right)$, for each $i \in \mathbb{I}$.

2. Applying Lemma 3 to $\left\{K^{i}\right\}_{i \in \mathbb{I} \backslash\{I\}}$ in (7), there is a predictable process of bounded variation $S^{2}$ starting in zero with $S^{2} \in L^{1}(\mathcal{P})$ and predictable $S^{2}$-integrable processes $\left\{\theta^{i, 2}\right\}_{i \in \mathbb{I} \backslash\{I\}}$, such that

$$
-K_{t}^{i}=\int_{0}^{t} \theta_{r}^{i, 2} \mathrm{~d} S_{r}^{2} \quad \mathcal{Q} \text {-a.e. }
$$

\footnotetext{
${ }^{49}$ Note that the asset price $S^{2}$ depends heavily on the equilibrium net trades.
} 
From this we can reformulate the bounded variation part in (5) and get $-K_{t}^{i}=$ $\int_{0}^{t} \theta_{r}^{i, 2} \mathrm{~d} S_{r}^{2}$, for each $i \in \mathbb{I} \backslash\{I\}$. Fix the following trading process for the riskless security $S^{0}$ for agent $i \in \mathbb{I} \backslash\{I\}$ :

$$
\theta_{t}^{i, 0}=\mathrm{E}^{Q}\left[\xi_{i}\right]+\int_{0}^{t} \theta_{r}^{i, 1} \mathrm{~d} S_{r}^{1}+\int_{0}^{t} \theta_{r}^{i, 2} \mathrm{~d} S_{t}^{2}-\theta_{t}^{i, 1} S_{t}^{1}-\theta_{t}^{i, 2} S_{t}^{2}, \quad \mathcal{Q} \text {-q.e. }
$$

where $\mathbb{E}^{\mathcal{Q}}\left[\xi_{i}\right]=\mathrm{E}^{Q}\left[\xi_{i}\right]$ for some Arrow-Debreu equilibrium pricing measure $Q \in$ $\mathcal{Q}\left(\xi_{i}\right) \cap \mathcal{Q}_{E} \neq \emptyset$. Clearly, $\int \theta^{i, 0} \mathrm{~d} S^{0} \equiv 0$ is a well defined square integrable integral and $\mathrm{E}^{Q}\left[\xi_{i}\right]=\left\langle\theta_{0}^{i}, S_{0}\right\rangle$. Predictability of $\theta^{i, 0}$ can easily be verified.

Substitution of the integral equations yields the self-financing property for $\theta^{i}$ :

$$
\left\langle\theta_{t}^{i}, S_{t}\right\rangle=\left\langle\theta_{0}^{i}, S_{0}\right\rangle+\int_{0}^{t}\left\langle\theta_{r}^{i}, \mathrm{~d} S_{r}\right\rangle \quad \mathcal{Q} \text {-q.e. }
$$

It follows that each trading strategy is admissible, i.e. $\theta^{i} \in \Theta(S)$, for each $i=\mathbb{I} \backslash\{I\}$. We observe via the self-financing property

$$
\begin{aligned}
\left\langle\theta_{T}^{i}, S_{T}\right\rangle+E_{i} & =\left(\theta_{T}^{i, 0}, \theta_{T}^{i, 1}, \theta_{T}^{i, 2}\right)^{\top}\left(D^{0}, D^{1}, D^{2}\right)+E_{i}=\bar{C}_{i}, \quad \text { Q-q.s. } \\
\left\langle\theta_{0}^{i}, S_{0}\right\rangle & =\mathbb{E}^{\mathcal{Q}}\left[\xi_{i}\right]=\mathrm{E}^{Q}\left[\xi_{i}\right]=\mathrm{E}^{Q}\left[\bar{C}_{i}-E_{i}\right]=\pi\left(e_{i}-\bar{c}_{i}\right) .
\end{aligned}
$$

Hence, each agent $i=\mathbb{I} \backslash\{I\}$ consumes $\left(\bar{c}_{i}, \bar{C}_{i}\right)$ via the portfolio strategy $\theta^{i}$.

3. In order to meet the market clearing condition in the Radner economy, consider the last agent $I \in \mathbb{I}$, equipped with $\theta^{I}=-\sum_{j \in \mathbb{I} \backslash\{I\}} \theta^{j}$, which guarantees market clearing, by the linear structure of $\Theta(S)$. The self-financing condition $\theta_{I} \in \Theta(S)$ holds by construction. We derive again by the Arrow-Debreu budget constraint, since $Q \in \mathcal{Q}_{E}$

$$
\left\langle\theta_{0}^{I}, S_{0}\right\rangle=\mathrm{E}^{Q}\left[-\sum_{j \in \mathbb{I} \backslash\{I\}} \xi_{j}\right]=\mathrm{E}^{Q}\left[\xi_{I}\right]=\pi\left(e_{I}-\bar{c}_{I}\right) .
$$

By the clearing condition of the Arrow-Debreu equilibrium we derive

$$
\xi_{I}=-\sum_{j \in \mathbb{I} \backslash\{I\}} \xi_{j}=\left\langle-\sum_{j \in \mathbb{I} \backslash\{I\}} \theta_{T}^{j}, S_{T}\right\rangle=-\sum_{j \in \mathbb{I} \backslash\{I\}}\left(\theta_{T}^{j, 0} S_{T}^{0}+\theta_{T}^{j, 1} S_{T}^{1}+\theta_{T}^{j, 2} S_{T}^{2}\right),
$$

which gives us the clearing condition in the Radner economy.

4. In the last step we show the individual optimality of the trading strategies. Suppose there is an agent $k \in \mathbb{I}$ capable of achieving a strictly preferred bundle $(c, C) \succ_{k}\left(\bar{c}_{k}, E_{k}+\xi_{k}\right)$ in terms of a different trading strategy $\theta^{C} \in \Theta(S)$. The Arrow-Debreu price system (at time $T$ ) in Theorem 2 satisfies $\Pi \in L^{1}(\mathcal{P})_{\oplus}^{*}$, the value of $(c, C)$ should be strictly higher in comparison to $\left(\bar{c}_{k}, \bar{C}_{k}\right)$, since preferences are semi-strictly monotone 50 This means

$$
\pi c+\mathrm{E}^{Q}[C]>\pi \bar{c}_{k}+\mathrm{E}^{Q}\left[E_{k}+\xi_{k}\right], \quad \text { for some } Q \in \mathcal{Q}_{E} .
$$

\footnotetext{
${ }^{50}$ In this argument, we benefit from the semi-strict positivity of the price system.
} 
Applying the Radner budget constraint for $(c, C)$, we have

$$
\pi e_{k}-\left\langle\theta_{0}^{C}, S_{0}\right\rangle+\mathrm{E}^{Q}\left[E_{k}+\left\langle\theta_{0}^{C}, S_{0}\right\rangle+\int_{0}^{T}\left\langle\theta_{t}^{C}, \mathrm{~d} S_{t}\right\rangle\right]>\pi \bar{c}_{k}+\mathrm{E}^{Q}\left[E_{k}+\xi_{k}\right],
$$

for some $Q \in \mathcal{Q}_{E}$. Since $\int \theta^{C, 0} \mathrm{~d} S^{0} \equiv 0$ and the stochastic integral $\int_{0}^{t} \theta_{r}^{C, 1} \mathrm{~d} S_{r}^{1}$ is a symmetric $\mathcal{Q}$-martingale, and hence a $Q$-martingale for every $Q \in \mathcal{Q}$ as well. By the market clearing and Lemma 3, $K_{T}^{C}=\int_{0}^{T} \theta_{r}^{C, 2} \mathrm{~d} S_{r}^{2}$ holds $\mathcal{Q}$-q.s. and since $-K^{C}$ is a $\mathcal{Q}$-martingale starting in zero, we conclude

$$
\pi e_{k}+\mathrm{E}^{Q}\left[E_{k}\right]=\pi e_{k}+\mathrm{E}^{Q}\left[E_{k}-K_{T}^{Q, C}\right]>\pi \bar{c}_{k}+\mathrm{E}^{Q}\left[E_{k}+\xi_{k}\right]
$$

This implies $0>\pi\left(\bar{c}_{k}-e_{k}\right)+\mathrm{E}^{Q}\left[\bar{C}_{k}-E_{k}\right]$, and contradicts the given Arrow-Debreu budget optimality of $\left(\bar{c}_{k}, \bar{C}_{k}\right)$.

This proves the existence of the Radner equilibrium. The properties of the equilibrium follow directly from the construction.

By $\mathcal{P}(\mathbb{F})$, we denote the predictable $\sigma$-algebra on $\bar{\Omega}=[0, T] \times \Omega$ with respect to the filtration $\mathcal{F}$ in Subsection 4.1. In the proof of Theorem 3, we applied the the following result.

Lemma 3 Fix a finite set $\left\{K^{i}\right\}_{i \in \mathbb{I}}$ of predictable, nondecreasing processes, starting in zero with $K_{T}^{i} \in L^{1}(\mathcal{P})$, then there is a predictable, nondecreasing process $S$, starting in zero with $S_{T} \in L^{1}(\mathcal{P})$ and a set $\left(\eta^{i}\right)_{i \in \mathbb{I}}$ of predictable and $S$-integrable processes such that

$$
K_{t}^{i}=\int_{0}^{t} \eta_{r}^{i} \mathrm{~d} S_{r} \quad \mathcal{P} \text {-q.e. } \quad \text { for every } i \in \mathbb{I} .
$$

Proof of Lemma 3 Set $K^{i, P}=K^{i}$ as a process on $(\Omega, \mathcal{B}(\Omega), P)$. By the properties of each $K^{i . P}$, there is a positive (random) measure $\mu^{i, P}$ on $(\bar{\Omega}, \mathcal{P}(\mathbb{F})$ ) satisfying

$$
A \mapsto \mu^{P}(A)=\mathrm{E}^{P}\left[\int_{0}^{T} 1_{A} \mathrm{~d} K_{t}^{i, P}\right], \quad A \in \mathcal{P}(\mathbb{F})
$$

The space of $\sigma$-finite signed measures $\mathcal{M}^{\sigma}(\bar{\Omega}, \mathcal{P}(\mathbb{F}))$ is a Banach lattic $£^{51}$ (see section IX.2 of Jacobs and Kurzweil (1978)), and especially a lattice group. By Proposition 5.1.12 of Constantinescu (1984), there is a finite family of strictly positive and $\sigma$-finite measures $\left(\nu_{\lambda}^{P}\right)_{\lambda \in \mathbb{L}} \subset \mathcal{M}^{\sigma}(\bar{\Omega}, \mathcal{P}(\mathbb{F}))$ such that

$$
\sum_{\lambda \in \mathbb{L}} \nu_{\lambda}^{P}=\mu^{P}=\bigvee_{i \in \mathbb{I}} \mu^{i, P} \in \mathcal{M}^{\sigma}(\bar{\Omega}, \mathcal{P}(\mathbb{F}))
$$

and for every $i \in \mathbb{I}$ there exists a subset $\mathbb{L}_{i} \subset \mathbb{L}$ with

$$
\mu^{i, P}=\sum_{\lambda \in \mathbb{L}_{i}} \nu_{\lambda}^{P} \in \mathcal{M}^{\sigma}(\bar{\Omega}, \mathcal{P}(\mathbb{F}))
$$

${ }^{51}$ Here, $\mathcal{M}^{\sigma}(\bar{\Omega}, \mathcal{P}(\mathbb{F}))$ is quipped with the natural ordering and the total variation norm. 
Absolute continuity follows, i.e. $\nu_{\lambda}^{P} \ll \mu^{P}$ for every $\lambda \in \mathbb{L}$. Hence, by the RadonNykodym theorem applied on $\left(\bar{\Omega}, \mathcal{P}(\mathbb{F}), \mu^{P}\right)$, we have

$$
\mathrm{d} \nu_{\lambda}^{P}=\frac{\mathrm{d} \nu_{\lambda}^{P}}{\mathrm{~d} \mu^{P}} \mathrm{~d} \mu^{P}, \quad \text { for every } \lambda \in \mathbb{L} .
$$

The density $\frac{\mathrm{d} \nu_{\lambda}^{P}}{\mathrm{~d} \mu^{P}}$ is in $L^{1}\left(\bar{\Omega}, \mathcal{P}(\mathbb{F}), \mu^{P}\right)$ if and only if $\nu_{\lambda}^{P}$ is $\sigma$-finite, and we have

$$
\mathrm{d} \mu^{i, P}=\sum_{\lambda \in \mathbb{L}_{i}} \mathrm{~d} \nu_{\lambda}^{P}=\sum_{\lambda \in \mathbb{L}_{i}} \frac{\mathrm{d} \nu_{\lambda}^{P}}{\mathrm{~d} \mu^{P}} \mathrm{~d} \mu^{P}=\eta^{i, P} \mathrm{~d} \mu^{P} .
$$

Similarly to the identification of $K^{i, P}$ via $\mu^{i, P}$, there is a predictable process $S^{P}$ with $S_{0}^{P}=0$ and increasing paths and a $\eta^{i, P} \in L^{1}\left(\bar{\Omega}, \mathcal{P}(\mathbb{F}), \mu^{P}\right)$, such that

$$
\mathrm{d} K_{t}^{i, P}=\eta_{t}^{i, P} \mathrm{~d} S_{t}^{P} \quad \text { for every } t \in[0, T] \text { and } i \in \mathbb{I} .
$$

In order to guarantee aggregating objects, i.e. $S=S^{P}$ and $\eta^{i}=\eta^{i, P} P \otimes \mathrm{d}$-a.e. for every $P \in \mathcal{P}$, we use the weak compactness of $\mathcal{P}$ in Assumption 1. The aggregation property holds by an application of Theorem 5.1 of Soner, Touzi, and Zhang (2012b), (see also Example 4.14 therein). The result follows.

\section{References}

Abramovich, Y., and C. Aliprantis (2002): An invitation to operator theory. American Mathematical Society.

Aliprantis, C., And K. Border (2006): Infinite dimensional analysis: a hitchhiker's guide. Springer Verlag.

Aliprantis, C., D. Brown, and O. Burkinshaw (1990): Existence and optimality of competitive equilibria. Springer Verlag.

Anderson, R., and R. Raimondo (2008): "Equilibrium in Continuous-Time Financial Markets: Endogenously Dynamically Complete Markets," Econometrica, 76(4), 841-907.

BASAK, S., And D. CuOco (1998): "An equilibrium model with restricted stock market participation," Review of Financial Studies, 11(2), 309.

Beissner, P. (2011): "Existence of Arrow-Debreu Equilibrium with Generalized Stochastic Differential Utility," Working Papers.

(2012): "Coherent Price Systems and Uncertainty-Neutral Valuation," Arxiv preprint arXiv:1202.6632.

Bewley, T. (2002): "Knightian decision theory. Part I," Decisions in economics and finance, 25(2), 79-110. 
Biagini, S., And M. Frittelli (2010): "On the Extension of the Namioka-Klee Theorem and on the Fatou Property for Risk Measures," Optimality and RiskModern Trends in Mathematical Finance, pp. 1-28.

Bion-Nadal, J., And M. Kervarec (2012): "Risk Measuring under Model Uncertainty," The Annals of Applied Probability, 22(1), 213-238.

Chateauneuf, A. (1991): "On the use of capacities in modeling uncertainty aversion and risk aversion," Journal of Mathematical Economics, 20(4), 343-369.

Chen, Z., And L. Epstein (2002): "Ambiguity, Risk, and Asset Returns in Continuous Time," Econometrica, 70(4), 1403-1443.

Cheridito, P., H. M. Soner, N. Touzi, And N. Victoir (2007): "Secondorder backward stochastic differential equations and fully nonlinear parabolic PDEs," Communications on Pure and Applied Mathematics, 60(7), 1081-1110.

Clarke, F. (1990): Optimization and nonsmooth analysis, vol. 5. Society for Industrial Mathematics.

Cohen, S. (2011): "Quasi-sure analysis, aggregation and dual representations of sublinear expectations in general spaces," Arxiv preprint arXiv:1110.2592.

Cohen, S., S. Ji, And S. Peng (2011): "Sublinear Expectations and Martingales in Discrete Time," Arxiv preprint arXiv:1104.5390.

Constantinescu, C. (1984): Spaces of measures, vol. 4. de Gruyter.

DANA, R. (1993): "Existence and uniqueness of equilibria when preferences are additively separable," Econometrica, 61(4), 953-957.

- (2004): "Ambiguity, uncertainty aversion and equilibrium welfare," Economic Theory, 23(3), 569-587.

Dana, R., And C. Le VAn (2010): "Overlapping Risk Adjusted Sets of Priors and the Existence of Efficient Allocations and Equilibria with Short-Selling," Journal of Economic Theory, 145(6), 2186-2202.

Dana, R., And M. Pontier (1992): "On existence of an Arrow-Radner equilibrium in the case of complete markets. A remark," Mathematics of Operations research, 17(1), 148-163.

DANA, R., ANd F. Riedel (2013): "Intertemporal Equilibria with Knightian Uncertainty," Journal of Economic Theory, 148(1), 1582-1605.

DANA, R.-A. (2002): "On equilibria when agents have multiple priors," Annals of Operations Research, 114(1), 105-115.

De Castro, L., and A. Chateauneuf (2011): "Ambiguity aversion and trade," Economic Theory, 48(2), 243-273. 
Debreu, G. (1959): Theory of value: An axiomatic analysis of economic equilibrium, vol. 17. New Haven: Yale University Press, c1959, 1973 printing.

Dellacherie, C., And P. Meyer (1978): "Probability and potential," Paris: Hermann.

Denis, L., M. Hu, and S. Peng (2011): "Function Spaces and Capacity Related to a Sublinear Expectation: Application to G-Brownian Motion Paths," Potential Analysis, 34(2), 139-161.

Denis, L., And M. Kervarec (2013): "Utility functions and optimal investment in non-dominated models," SIAM J. Control Optim., 51(3), 1803-1822.

Dow, J., And S. DA Costa Werlang (1992): "Uncertainty aversion, risk aversion, and the optimal choice of portfolio," Econometrica, 60(1), 197-204.

Duffie, D. (1986): "Stochastic Equilibria: Existence, Spanning Number, and the No Expected Financial Gain from Trade'Hypothesis," Econometrica, 54(5), $1161-1184$.

Duffie, D., And C. HuAng (1985): "Implementing Arrow-Debreu equilibria by continuous trading of few long-lived securities," Econometrica, 53(6), 1337-1356.

Duffie, D., And W. Shafer (1985): "Equilibrium in incomplete markets: I:: A basic model of generic existence," Journal of Mathematical Economics, 14(3), $285-300$.

Duffie, D., And W. Zame (1989): "The consumption-based capital asset pricing model," Econometrica, 57(6), 1279-1297.

Epstein, L., And S. Ji (2013a): "Ambiguous volatility and asset pricing in continuous time," To Appear: Rev. Finan. Stud.

Epstein, L., And S. Ji (2013b): "Ambiguous volatility, possibility and utility in continuous time," arXiv preprint arXiv:1103.1652v\%.

Epstein, L., And M. Schneider (2003): "Recursive multiple-priors," Journal of Economic Theory, 113(1), 1-31.

Epstein, L., And T. WAng (1994): "Intertemporal Asset Pricing under Knightian Uncertainty," Econometrica: Journal of the Econometric Society, 62(3), 283-322.

Epstein, L., AND J. ZhANG (2001): "Subjective probabilities on subjectively unambiguous events," Econometrica, 69(2), 265-306.

FARO, J. (2009): "Variational bewley preferences," Discussion paper, Technical report, Cedeplar-FACE-UFMG.

Feyel, D., And A. De La Pradelle (1977): "Topologies fines et compactifications associées à certains espaces de Dirichlet," in Annales de l'institut Fourier, vol. 27, pp. 121-146. Institut Fourier. 
Feyel, D., And A. De La Pradelle (1989): "Espaces de Sobolev gaussiens," in Annales de l'institut Fourier, vol. 39, pp. 875-908. Institut Fourier.

GilboA, I., And D. Schmeidler (1989): "Maxmin expected Utility with NonUnique Prior," Journal of Mathematical Economics, 18(2), 141-153.

Hansen, L., And T. Sargent (2001): "Robust control and model uncertainty," American Economic Review, 91(2), 60-66.

Hara, C., J. Huang, and C. Kuzmics (2007): "Representative consumer's risk aversion and efficient risk-sharing rules," Journal of Economic Theory, 137(1), $652-672$.

HerzberG, F., And F. Riedel (2013): "Existence of financial equilibria in continuous time with potentially complete markets," To Appear: Journal of Mathematical Economics.

HuAng, C. (1985): "Information structure and equilibrium asset prices," Journal of Economic Theory, 35(1), 33-71.

(1987): "An intertemporal general equilibrium asset pricing model: The case of diffusion information," Econometrica, 55(1), 117-42.

Huber, P., And V. Strassen (1973): "Minimax Tests and the Neyman-Pearson Lemma for Capacities," The Annals of Statistics, 1(2), 251-263.

Hugonnier, J., S. Malamud, and E. Trubowitz (2012): "Endogenous Completeness of Diffusion Driven Equilibrium Markets," Econometrica, 80(3), 1249 1270 .

Jacobs, K., And J. KurzweIL (1978): Measure and integral. Academic Press New York.

Karatzas, I., J. Lehoczky, and S. Shreve (1990): "Existence and uniqueness of multi-agent equilibrium in a stochastic, dynamic consumption/investment model," Mathematics of Operations research, 15(1), 80-128.

Kervarec, M. (2008): "Etude des modeles non domines en mathematiques financieres," These de Doctorat en Mathematiques, Universite d' Evry.

Kreps, D. (1982): Multiperiod securities and the efficient allocation of risk: $A$ comment on the Black-Scholes option pricing model. University of Chicago Press.

LAUREnt, P. (1972): Approximation et optimisation, vol. 2. Université Scientifique et Médicale de Grenoble.

Maccheroni, F., M. Marinacci, and A. Rustichini (2006): "Ambiguity Aversion, Robustness, and the Variational Representation of Preferences," Econometrica, 74(6), 1447-1498. 
Magill, M., And M. QuinziI (2002): Theory of incomplete markets, vol. 1. Mit press.

Martins-da Rocha, V., And F. Riedel (2010): "On equilibrium prices in continuous time," Journal of Economic Theory, 145(3), 1086-1112.

MukerJi, S., AND J. TAllon (2001): "Ambiguity aversion and incompleteness of financial markets," Review of Economic Studies, 68(4), 883-904.

Nutz, M., And H. Soner (2012): "Superhedging and Dynamic Risk Measures under Volatility Uncertainty," To appear in SIAM J. Control Optim.

PenG, S. (2006): "G-expectation, G-Brownian Motion and Related Stochastic Calculus of Itô Type," Stochastic analysis and applications, The Abel Symposium $2005,541-567$.

- (2007): "G-Brownian Motion and Dynamic Risk Measure under Volatility Uncertainty," Arxiv preprint arXiv:0711.2834.

— (2010): "Nonlinear Expectations and Stochastic Calculus under Uncertainty," Arxiv preprint ArXiv:1002.4546.

Peng, S., Y. Song, and J. Zhang (2013): "A complete representation theorem for G-martingales," arXiv preprint arXiv:1201.2629v2.

Pham, T., And J. Zhang (2012): "Some Norm Estimates for Semimartingales," Arxiv preprint arXiv:110\%.4020v2.

RADNER, R. (1972): "Existence of equilibrium of plans, prices, and price expectations in a sequence of markets," Econometrica: Journal of the Econometric Society, pp. 289-303.

Ravanelli, C., And G. Svindland (2013): "Comonotone Pareto optimal allocations for law invariant robust utilities on $L^{1}$," To Appear: Finance and Stochastics.

RiEDEL, F. (2009): "Optimal stopping with multiple priors," Econometrica, 77(3), $857-908$.

Rigotti, L., And C. Shannon (2012): "Sharing risk and ambiguity," Journal of Economic Theory, 147(5), 2028-2039.

Soner, H. M., N. Touzi, And J. Zhang (2012a): "Wellposedness of second order backward SDEs," Probability Theory and Related Fields, 153(1-2), 149-190.

Soner, M., N. Touzi, and J. Zhang (2012b): "Quasi-sure Stochastic Analysis through Aggregation," Electronic Journal of Probability, 16, 1844-1879.

VORBRINK, J. (2010): "Financial markets with volatility uncertainty," arXiv preprint arXiv:1012.1535. 
XU, Y. (2010): "Backward stochastic differential equations under super linear Gexpectation and associated Hamilton-Jacobi-Bellman equations," Arxiv preprint arXiv:1009.1042.

Yannelis, N. C. (1991): "The Core of an Economy with Differential Information," Economic Theory, 1(2), 183-97.

ZEIDleR, E. (1985): Nonlinear Functional Analysis: Variational Methods and Optimization. Springer. 